\title{
Building spectral element dynamic matrices using finite element models of waveguide slices and elastodynamic equations
}

\author{
P.B. Silva*, A.L. Goldstein and J.R.F. Arruda \\ Departamento de Mecânica Computacional, FEM, Universidade Estadual de Campinas. R. Mendeleyev, \\ Campinas - SP, Brazil
}

Received 25 September 2012

Revised 6 November 2012

Accepted 9 November 2012

\begin{abstract}
Structural spectral elements are formulated using the analytical solution of the applicable elastodynamic equations and, therefore, mesh refinement is not needed to analyze high frequency behavior provided the elastodynamic equations used remain valid. However, for modeling complex structures, standard spectral elements require long and cumbersome analytical formulation. In this work, a method to build spectral finite elements from a finite element model of a slice of a structural waveguide (a structure with one dimension much larger than the other two) is proposed. First, the transfer matrix of the structural waveguide is obtained from the finite element model of a thin slice. Then, the wavenumbers and wave propagation modes are obtained from the transfer matrix and used to build the spectral element matrix. These spectral elements can be used to model homogeneous waveguides with constant cross section over long spans without the need of refining the finite element mesh along the waveguide. As an illustrating example, spectral elements are derived for straight uniform rods and beams and used to calculate the forced response in the longitudinal and transverse directions. Results obtained with the spectral element formulation are shown to agree well with results obtained with a finite element model of the whole beam. The proposed approach can be used to generate spectral elements of waveguides of arbitrary cross section and, potentially, of arbitrary order.
\end{abstract}

Keywords: Spectral elements, finite elements, waveguides, propagation modes, higher-order modes, mid frequency, structural dynamics

\section{Introduction}

Numerous hybrid waveguide finite element methods have been developed in recent years. These methods were motivated by the need to minimize the computational cost required to analyze long waveguides with complex cross sections in mid frequencies. A semi-analytical approach was proposed in 1972 by Dong and Nelson [8] for studying laminated orthotropic plates exhibiting a plane-strain behavior. In this case, the displacement field was described by two infinite sets of waves traveling in opposite senses and an interpolation function over each lamina thickness. Two years later, Aalami [1] extended the previous work and analyzed three-dimensional prismatic guides using a two-dimensional interpolation function to describe the displacement field in a bar with arbitrary cross section. More recently, Gavric [11,12] used a similar approach to obtain dispersion properties of thin-walled beams and a free rail. The strategy used by Gavric in his works has been applied by many researchers to compute frequency and time

*Corresponding author: P.B. Silva, Departamento de Mecânica Computacional, FEM, Universidade Estadual de Campinas. R. Mendeleyev, 200, 13083-960, Campinas - SP, Brazil. Tel.: +55 193521 3395; Fax: +55 193289 3722; E-mail: pbrandaos@ fem.unicamp.br. 
domain transient responses of elastic and viscoelastic waveguides [16,18], or was associated with the classical finite element method to study the interaction of guided waves with non-axisymmetric damage [5]. This method is usually known as the Semi-Analytical Finite Element (SAFE) method. Finnveden [10] has applied a similar technique, but using higher order polynomial functions for the cross section discretization.

More recently, a method was developed where a waveguide model can be derived from a standard FE code used to model a slice of the waveguide. The wave model can be used to compute the spectral relations, the group and energy velocities, and the forced response [17,21]. These wave approaches that use a finite element model of a slice of the waveguide are based on the periodic structure theory developed by Mead [19] in the early seventies. Waki and collaborators [24] have investigated numerical issues of this method based on a previous work by Zhong and Williams [25]. The so-called Wave and Finite Element (WFE) method was applied to describe the low and mid frequency dynamic behavior of structures [20]. Compared to the semi-analytical methods previously commented, the application of the WFE method is much more straightforward, as it does not need an ad hoc analytical formulation.

The method proposed here is based on the WFE method. The basic idea consists of using the WFE method to build a spectral element, as defined by Doyle [9]. A spectral element (or continuous element) uses the analytical, exact interpolation functions to obtain the dynamic stiffness matrix of a structure. However, for modeling complex structures, standard spectral elements require long and cumbersome analytical formulation. The present work shows that it is possible to build a spectral element matrix for a structure with arbitrary cross section and arbitrary length from the wave properties computed from a thin slice of the structure modeled with conventional finite elements.

The use of the WFE method for computing the wavenumbers and wave propagation modes is reviewed in Section 2. In Section 3, the proposed method is formulated and applied to derive numerical spectral elements using elementary rod and beams theories from a structural waveguide slice modeled with solid finite elements. The proposed method will be called here "Wave Spectral Finite Element Method" (WSFEM). It was originally applied to a straight rod problem in [3]. In the present work, the method is extended to derive spectral elements that can be used to compute the dynamic forced response of a beam subjected to different load conditions including a uniform longitudinal force distribution, transverse forces, and non-uniform longitudinal (line) forces. Results are compared with results from a standard FE analysis, thus validating the method. The proposed method is applicable to more complex structures such as car tires [6,7] and ducts. Moreover, because the spectral dynamic matrix is obtained, instead of the standard wave propagation solution, the proposed spectral elements can be easily combined with standard finite elements using a mobility approach.

\section{Finite element analysis of periodic structures}

Consider a structural waveguide represented as a finite or infinite number of cells as shown in Fig. 1. Assume that a single cell representing a short slice from the structural waveguide is modeled with solid structural finite elements meshed with an equal number of nodes on the left and right sides (Fig. 1).

\subsection{Dynamic stiffness matrix of a waveguide slice}

The dynamic stiffness matrix of the slice can be obtained by taking the Fourier transform of the equations of motion:

$$
\left[\mathbf{K}+i \omega \mathbf{C}-\omega^{2} \mathbf{M}\right] \mathbf{q}=\mathbf{F}
$$

where $\mathbf{K}, \mathbf{M}$, and $\mathbf{C}$ are the stiffness, mass and damping matrices, respectively, $\mathbf{q}$ is the vector of the displacement degrees of freedom and $\mathbf{F}$ the applied forces. The dynamic stiffness matrix is defined as:

$$
\hat{\mathbf{D}}=\left[\mathbf{K}+i \omega \mathbf{C}-\omega^{2} \mathbf{M}\right]
$$

Separating the right $(R)$ and left $(L)$ side degrees of freedom and interior $(I)$ degrees of freedom (DOF) and assuming there are no forces applied to the interior nodes, the partitioned matrix can be written as:

$$
\left[\begin{array}{ccc}
\hat{D}_{I I} & \hat{D}_{I L} & \hat{D}_{I R} \\
\hat{D}_{L I} & \hat{D}_{L L} & \hat{D}_{L R} \\
\hat{D}_{R I} & \hat{D}_{R L} & \hat{D}_{R R}
\end{array}\right]\left\{\begin{array}{c}
q_{I} \\
q_{L} \\
q_{R}
\end{array}\right\}=\left\{\begin{array}{c}
0 \\
F_{L} \\
F_{R}
\end{array}\right\}
$$




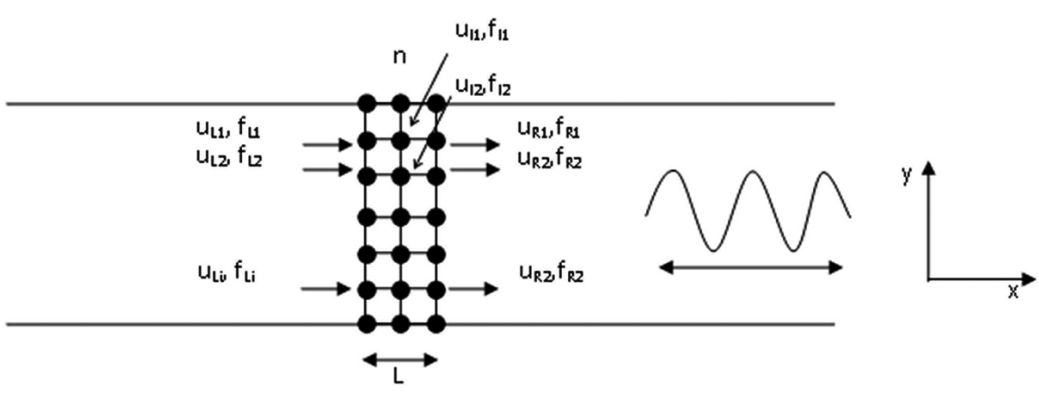

Fig. 1. Finite element mesh of waveguide cross section.
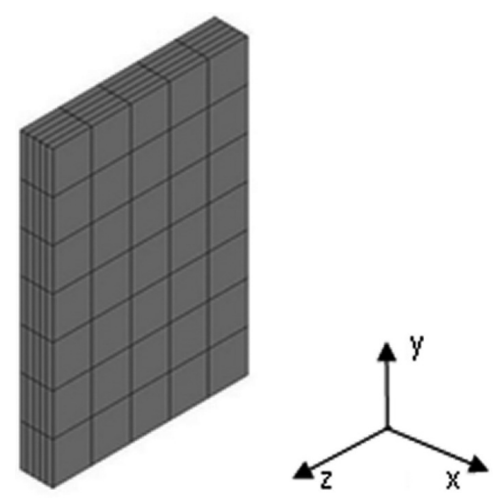

Fig. 2. Finite element mesh of beam slice modeled with SOLID45 elements using commercial FE code ANSYS ${ }^{\circledR}$.

The interior degrees of freedom $\mathbf{q}_{\mathrm{I}}$ of the dynamic stiffness matrix can be condensed using the first row of the Eq. (2):

$$
\mathbf{q}_{\mathrm{I}}=-\hat{\mathbf{D}}_{\mathrm{II}}^{-1}\left(\hat{\mathbf{D}}_{\mathrm{IL}} \mathbf{q L}_{\mathrm{L}}+\hat{\mathbf{D}}_{\mathrm{IR}} \mathbf{q R}_{\mathrm{R}}\right)
$$

Using this expression, the new condensed dynamic matrix, after elimination of the interior DOF, can be written as:

$$
\left[\begin{array}{ll}
\mathbf{D}_{\mathrm{LL}} & \mathrm{D}_{\mathrm{LR}} \\
\mathrm{D}_{\mathrm{RL}} & \mathrm{D}_{\mathrm{RR}}
\end{array}\right]\left\{\begin{array}{l}
\mathrm{q}_{\mathrm{L}} \\
\mathrm{q}_{\mathrm{R}}
\end{array}\right\}=\left\{\begin{array}{l}
\mathbf{F}_{\mathrm{L}} \\
\mathbf{F}_{\mathrm{R}}
\end{array}\right\}
$$

where:

$$
\begin{aligned}
& \mathbf{D}_{L L}=\hat{\mathbf{D}}_{\mathrm{LL}}-\hat{\mathbf{D}}_{\mathrm{LI}} \hat{\mathbf{D}}_{\mathrm{II}}^{-1} \hat{\mathbf{D}}_{\mathrm{IL}}, \mathbf{D}_{\mathrm{RL}}=\hat{\mathbf{D}}_{\mathrm{RL}}-\hat{\mathbf{D}}_{\mathrm{RI}} \hat{\mathbf{D}}_{\mathrm{II}}^{-1} \hat{\mathbf{D}}_{\mathrm{IL}} \\
& \mathbf{D}_{\mathrm{LR}}=\hat{\mathbf{D}}_{\mathrm{LR}}-\hat{\mathbf{D}}_{\mathrm{LI}} \hat{\mathbf{D}}_{\mathrm{II}}^{-1} \hat{\mathbf{D}}_{\mathrm{IR}}, \mathbf{D}_{\mathrm{RR}}=\hat{\mathbf{D}}_{\mathrm{RR}}-\hat{\mathbf{D}}_{\mathrm{RI}} \hat{\mathbf{D}}_{\mathrm{II}}^{-1} \hat{\mathbf{D}}_{\mathrm{IR}}
\end{aligned}
$$

The partitioned matrix on the left side of Eq. (3) depends only on the dynamic stiffness matrix of a section of the waveguide, and it will be used as the basis for the analysis that follows.

\subsection{Transfer matrix formulation}

The transfer matrix method has been extensively used to solve frame structures and rotor system dynamic problems [22]. Instead of relating external forces and displacements at the left and right faces of an element, the transfer matrix relates internal forces and displacements at one side to the forces and displacements at the other side. In addition, instead of using the direct stiffness method to obtain the dynamic matrix for the whole structure, the state vector (displacements and internal forces) is propagated from one end of the assembled structure to the other end. The boundary conditions are then applied generating the solution to the problem.

Referring to Fig. 1, and assuming there are no forces applied to the structure at the junction, one can write:

$$
\begin{aligned}
& \mathrm{q}_{\mathrm{L}}^{\mathrm{n}+\mathbf{1}}=\mathrm{q}_{\mathrm{R}}^{\mathrm{n}} \\
& \mathrm{f}_{\mathrm{L}}^{\mathrm{n}+\mathbf{1}}=-\mathrm{f}_{\mathrm{R}}^{\mathrm{n}}
\end{aligned}
$$

The transfer matrix $\mathbf{T}$ links the displacements and forces at the cross sections $n$ and $n+1$. Using the relations in Eqs (4) and (5) matrix $\mathbf{T}$ is defined as:

$$
\mathbf{T}\left\{\begin{array}{c}
\mathrm{q}_{\mathrm{L}}^{\mathrm{n}} \\
\mathrm{f}_{\mathrm{L}}^{\mathrm{n}}
\end{array}\right\}=\left\{\begin{array}{l}
\mathrm{q}_{\mathrm{R}}^{\mathrm{n}} \\
\mathrm{f}_{\mathrm{R}}^{\mathrm{n}}
\end{array}\right\}=\left\{\begin{array}{c}
\mathrm{q}_{\mathrm{L}}^{\mathrm{n}+1} \\
-\mathrm{f}_{\mathrm{L}}^{\mathrm{n}+1}
\end{array}\right\}
$$




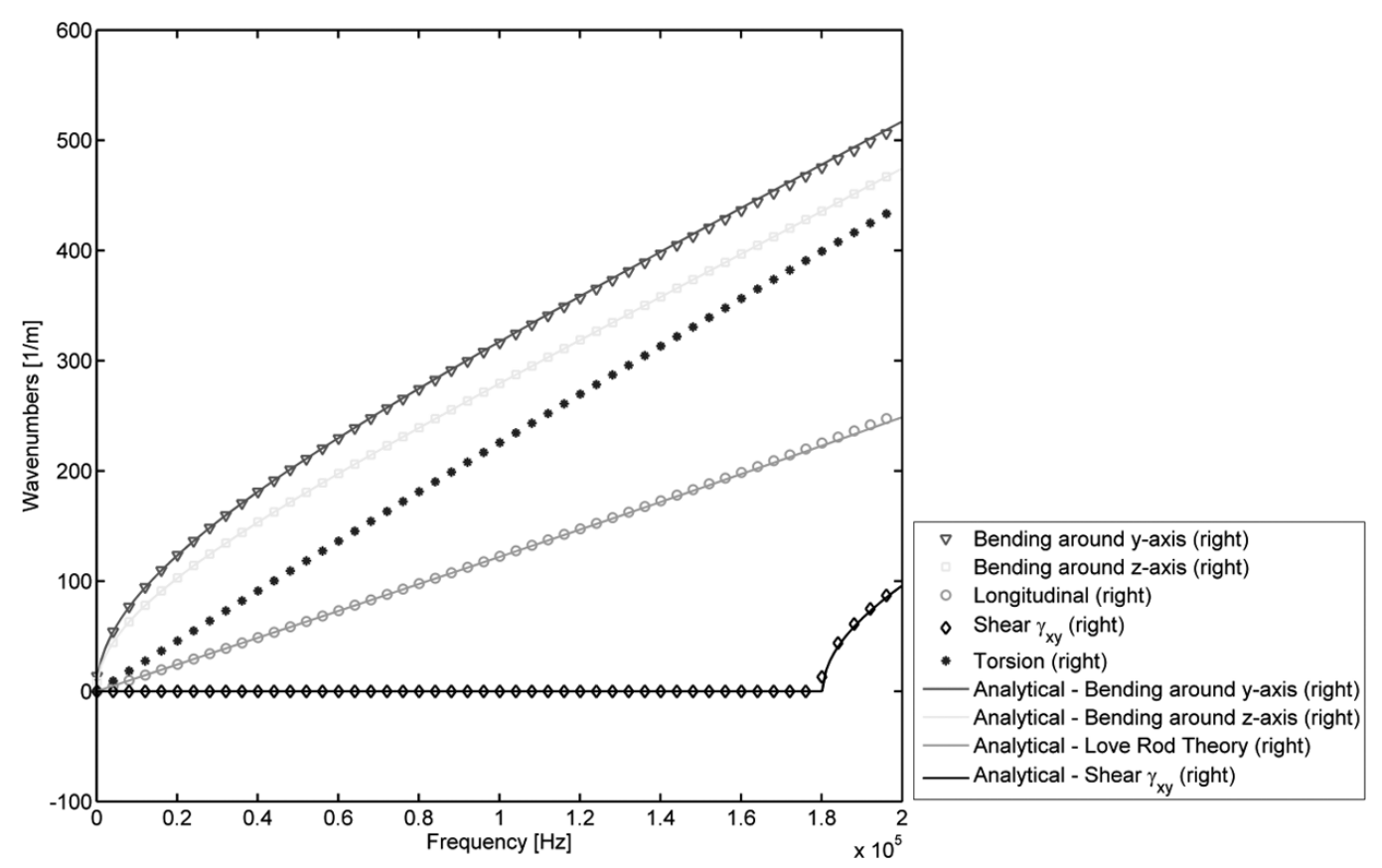

Fig. 3. Real part of the wavenumbers obtained from the transfer matrix of the beam slice modeled with solid finite elements.

Using the sign convention, the internal and external (capital F) forces are related as follows:

$$
\begin{aligned}
& \mathbf{F}_{\mathbf{L}}=\mathrm{f}_{\mathbf{L}} \\
& \mathbf{F}_{\mathbf{R}}=-\mathbf{f}_{\mathbf{R}}
\end{aligned}
$$

Using the first equation of the condensed dynamic stiffness matrix in Eqs (3), (7) and (8), it is possible to write:

$$
\mathrm{q}_{\mathrm{L}}^{\mathrm{n}+\mathbf{1}}=\mathrm{D}_{\mathrm{LR}}^{-\mathbf{1}}\left(\mathrm{f}_{\mathrm{L}}^{\mathrm{n}}-\mathrm{D}_{\mathrm{LL}} \mathbf{q}_{\mathrm{L}}^{\mathrm{n}}\right)
$$

Substituting Eq. (9) into the second row of Eq. (3) and using Eq. (5) gives:

$$
\left(D_{R L}-D_{R R} D_{L R}^{-1} D_{L L}\right) q_{L}^{n}+D_{R R} D_{L R}^{-1} f_{L}^{n}=f_{L}^{n+1}
$$

Now, using Eqs (9) and (10), the transfer matrix can be written as:

$$
T=\left[\begin{array}{cc}
-D_{L R}^{-1} D_{L L} & D_{L R}^{-1} \\
-D_{R L}+D_{R R} D_{L R}^{-1} D_{L L} & -D_{R R} D_{L R}^{-1}
\end{array}\right]
$$

From the periodic structure theory developed by Mead [19], one can write the following relations between consecutive slices of the waveguide:

$$
\begin{aligned}
& \mathbf{q}_{\mathbf{L}}^{n+1}=\lambda \mathbf{q}_{\mathbf{L}}^{n} \\
& \mathbf{f}_{\mathbf{L}}^{n+1}=-\lambda \mathbf{f}_{\mathbf{L}}^{n}
\end{aligned}
$$

Substituting Eqs (12) and (13) into Eq. (6), the following eigenvalue problem can be written:

$$
\mathbf{T}\left\{\begin{array}{c}
\mathbf{q}_{\mathbf{L}} \\
\mathbf{f}_{\mathrm{L}}
\end{array}\right\}=\lambda\left\{\begin{array}{c}
\mathbf{q}_{\mathbf{L}} \\
\mathbf{f}_{\mathbf{L}}
\end{array}\right\}
$$




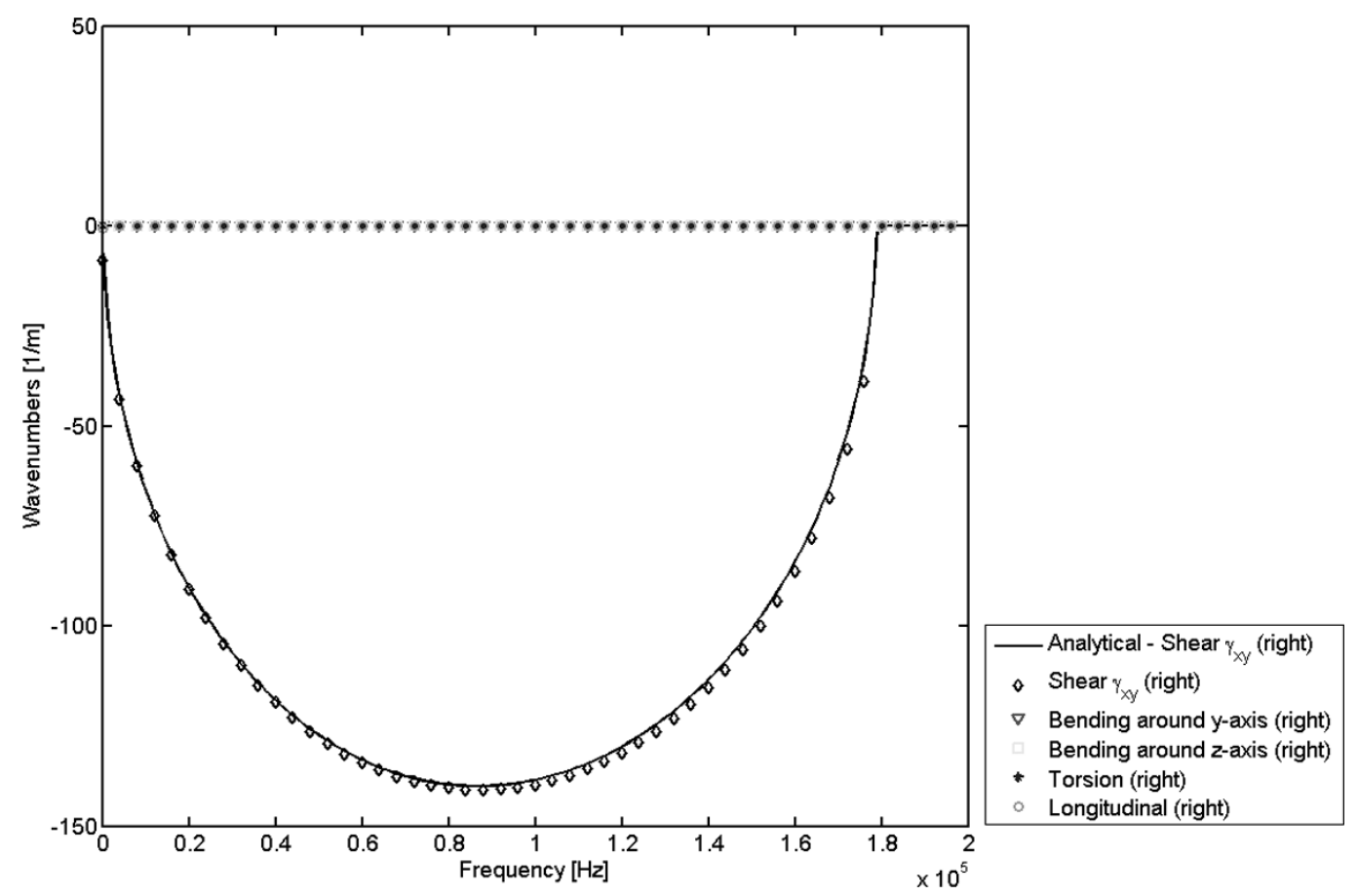

Fig. 4. Imaginary part of the wavenumbers obtained from the transfer matrix of the beam slice modeled with solid finite elements.

The eigenvalue problem yields $m$ independent complex conjugate eigenvalue pairs and corresponding wave propagation modes [14], where $m=n / 2$ is the number of degrees of freedom on each side of the slice. The eigenvectors $\boldsymbol{\Phi}=\left[\boldsymbol{\Phi}_{\mathbf{q}} ; \boldsymbol{\Phi}_{\mathbf{f}}\right]$ are of size $(2 m \times 2 m)$ where $\boldsymbol{\Phi}_{\mathbf{q}}$ are the $m$ displacement components and $\boldsymbol{\Phi}_{f}$ are the $m$ force components. Note that although, in general, the eigenvalues are complex, in the absence of damping, the amplitude of the propagating waves remains constant, which implies that the eigenvalues are such that $\left|\lambda_{j}\right|=1$ and $\lambda_{j}(\omega)=e^{-i k_{j} L}, i=\sqrt{-1}$.

Then, the wavenumbers can be obtained from the eigenvalues using:

$$
k_{j}(\omega)=-\frac{\ln \lambda_{j}(\omega)}{i L}
$$

The dependence upon frequency is shown to emphasize this feature of the wavenumbers and propagation modes.

There can be some difficulty in sorting the eigenvalues and tracking the frequency evolution of the wavenumbers. As a result, the eigenvalue curves cross over each other creating a physically unrealistic discontinuity in the dispersion curves. Therefore, it is necessary to process the sorting so that the smoothly varying eigenvalues can be tracked. This can be done by using the Modal Assurance Criterion (MAC), as proposed by Mencik [20]. It consists in estimating the correlation coefficient between wave propagation modes evaluated at small enough frequency steps $\Delta_{\omega}$. Thus, given two propagation mode vectors at adjacent frequencies $\omega_{k}$ and $\omega_{k+1}$, where $\omega_{k+1}=\omega_{k}+\Delta_{\omega}$, the MAC between the eigenvector at frequency $\omega_{n}$ and the eigenvector at frequency $\omega_{n+1}$ is calculated as:

$$
\frac{\Phi_{n}^{H}\left(\omega_{k}\right)}{\left\|\Phi_{n}\left(\omega_{k}\right)\right\|} \frac{\Phi_{n}\left(\omega_{k+1}\right)}{\left\|\Phi_{n}\left(\omega_{k+1}\right)\right\|}=\max _{m}\left\{\frac{\Phi_{n}^{H}\left(\omega_{k}\right)}{\left\|\Phi_{n}\left(\omega_{k}\right)\right\|} \frac{\Phi_{m}\left(\omega_{k+1}\right)}{\left\|\Phi_{m}\left(\omega_{k+1}\right)\right\|}\right\}
$$

Using this property, based on the hermitian scalar product, the eigenvectors can be properly sorted at each frequency and the corresponding eigenvalues can be tracked.

\section{Numerical example: Straight uniform beam}

For the numerical simulations presented in this section, a $1 \mathrm{~mm}$-long slice of a steel beam (Young's modulus $E=2.1 \times 10^{11} \mathrm{~Pa}$, density $\rho=7850 \mathrm{~kg} / \mathrm{m}^{3}$, Poisson's ratio $\left.v=0.3\right)$ with height $h=9 \mathrm{~mm}$ and width $b=6 \mathrm{~mm}$ 

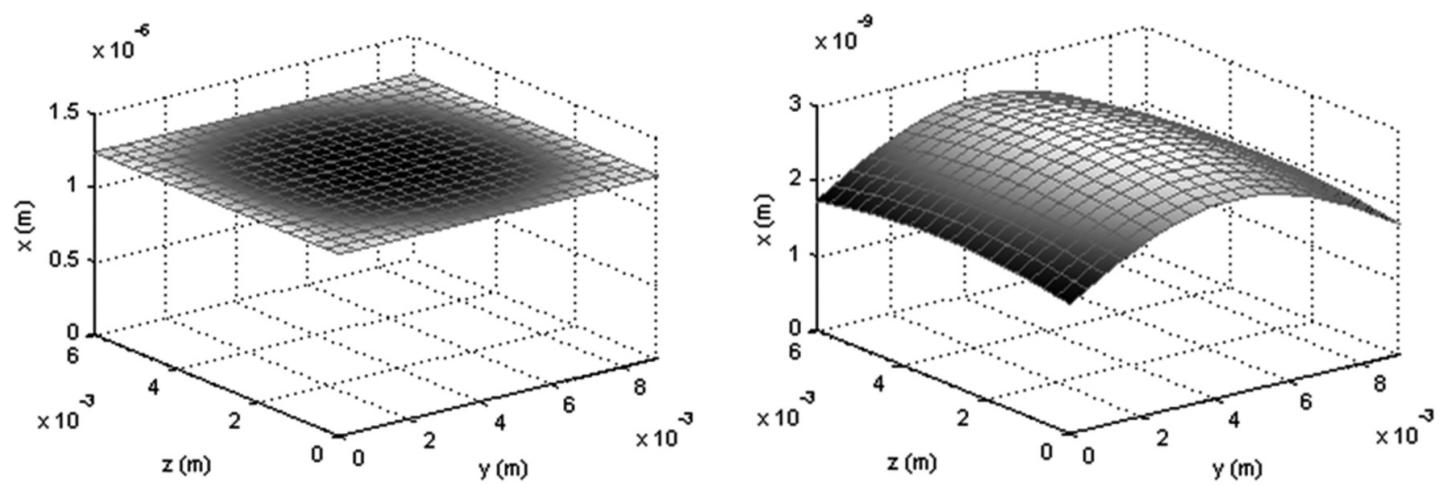

Fig. 5. Spatial representation of the wave mode corresponding to longitudinal wave propagation ( $x$ direction) at $1 \mathrm{kHz}$ (left) and $200 \mathrm{kHz}$ (right).

was modeled with solid finite elements [4] SOLID45 using ANSYS ${ }^{\circledR}$ (Fig. 2). The slice thickness was chosen to avoid spatial aliasing problems, as discussed in reference [15].

The mass and stiffness matrices of the beam slice were extracted from the finite element analysis and then postprocessed to assemble the transfer matrix according to Eq. (11). An eigenvalue analysis of the numerical transfer matrix $\mathbf{T}$ results in the wavenumbers and propagation modes of the beam. The real and imaginary parts of the wavenumbers are shown in Figs 3 and 4, respectively. The wavenumbers numerically obtained correspond to the longitudinal, torsional, bending and shear propagation modes of the beam. They match well the wavenumbers predicted by the classical rod and beam theories, as shown, for instance, in references [4,23]. For the sake of clarity, the analytical wavenumbers are also plotted in Figs 3 and 4. Figure 5 shows the spatial representation of the wave propagation mode corresponding to longitudinal waves ( $x$ direction) at $1 \mathrm{kHz}$ (left) and $200 \mathrm{kHz}$ (right). The distortion of the displacement field over the cross section at higher frequencies can be observed.

\subsection{Derivation of the numerical spectral element matrix for a rod}

The elementary theory considers the rod to be long and slender and assumes it supports only 1-D axial stress. In addition, lateral contraction (or the Poisson's ratio effect) is neglected. It has been shown previously by the authors [3] that is it possible to obtain the spectral element matrix of a rod using the transfer matrix built from the mass and stiffness matrices obtained from the finite element model of a slice and to reduce the displacement field so that the resulting number of degrees of freedom is the same as in the theoretical spectral matrix. In addition, in [2] it has been shown that it is possible to keep all the nodal DOF of the finite element model of the slice and build new numerical spectral elements for a rod with arbitrary cross section and length. In this sub-section these previous results are reviewed to illustrate the use of the proposed method.

First, it is assumed that the longitudinal displacements can be written as a superposition of propagation modes:

$$
\mathbf{u}_{\mathbf{x}}=\sum_{r} A_{r} \mathbf{\Phi}_{\mathbf{x r}} e^{-i k_{r} x}
$$

where $A_{r}$ are constants determined from the applied loads and boundary conditions, $k_{r}$ and $\boldsymbol{\Phi}_{\mathbf{x r}}$ are the wavenumbers and the longitudinal wave propagation modes that are obtained from the eigenvalue decomposition of the transfer matrix obtained from the finite element analysis, respectively. When analyzing the longitudinal behavior, only the eigenvector components associated with displacement degrees of freedom in the $x$-direction were used. Thus, the indices $x$ and $r$ in $\boldsymbol{\Phi}_{\mathbf{x r}}$ are used to designate the row corresponding to the longitudinal DOF and the columns corresponding to longitudinal wave propagation mode taken into account, respectively.

The axial strain due to the longitudinal deformation is then given by:

$$
\varepsilon_{\mathbf{x x}}=\frac{\partial \mathbf{u}_{\mathbf{x}}}{\partial x}=\sum_{r}-i k_{r} A_{r} \boldsymbol{\Phi}_{\mathbf{x r}} e^{-i k_{r} x}
$$


The expression for the longitudinal internal force is given by $f_{x}=-E A \varepsilon_{x x}$. Substituting Eq. (18) for the longitudinal strain into the expression of the force results in:

$$
\mathbf{f}_{\mathbf{x}}=-E S_{e} \sum_{r}-i k_{r} A_{r} \boldsymbol{\Phi}_{\mathbf{x r}} e^{-i k_{r} x}
$$

where $E$ is the Young's modulus and $S_{e}$ is the fraction of the total cross section area corresponding to each node assuming a uniform average over the number of nodes.

Using Eq. (17), the displacements at nodes located at $x=0$ and $x=L_{x}$, where $L_{x}$ is an arbitrary length of the element, can be written as a function of the wavenumbers and corresponding wave modes in matrix form, as:

$$
\left\{\begin{array}{c}
\mathbf{u}(x=0) \\
\mathbf{u}\left(x=L_{x}\right)
\end{array}\right\}=\left\{\begin{array}{c}
\mathbf{u}_{\mathbf{0}} \\
\mathbf{u}_{\mathbf{L}}
\end{array}\right\}=\left[\begin{array}{c}
\mathbf{\Phi}_{\mathbf{x}} \\
\mathbf{\Phi}_{\mathbf{x}} \boldsymbol{\Lambda}_{\mathbf{1}}^{\mathbf{R}}
\end{array}\right]\left\{\begin{array}{c}
A_{1} \\
A_{2}
\end{array}\right\}
$$

where $\boldsymbol{\Phi}_{\mathbf{x}}=\left[\begin{array}{ll}\boldsymbol{\Phi}_{\mathbf{x} 1} & \boldsymbol{\Phi}_{\mathbf{x} 2}\end{array}\right], \boldsymbol{\Lambda}_{1 \mathbf{L}}^{\mathbf{R}}=\left[\begin{array}{cc}e^{-i k_{1} L_{x}} & 0 \\ 0 & e^{-i k_{2} L_{x}}\end{array}\right], k_{1}$ and $k_{2}$ are the wavenumbers, $\boldsymbol{\Phi}_{\mathbf{x} 1}$ and $\boldsymbol{\Phi}_{\mathbf{x} 2}$ are the pair of wave propagation modes corresponding to longitudinal waves propagating along the rod in opposite senses.

In the same way, using Eqs (7), (8) and (19), one gets:

$$
\left\{\begin{array}{c}
\mathbf{F}(x=0) \\
\mathbf{F}\left(x=L_{x}\right)
\end{array}\right\}=\left\{\begin{array}{l}
\mathbf{F}_{\mathbf{0}} \\
\mathbf{F}_{\mathbf{L}}
\end{array}\right\}=E S_{e}\left[\begin{array}{r}
-\boldsymbol{\Phi}_{\mathbf{x}} \boldsymbol{\Lambda}_{20}^{\mathbf{R}} \\
\boldsymbol{\Phi}_{\mathbf{x}} \boldsymbol{\Lambda}_{\mathbf{2}}^{\mathbf{R}}
\end{array}\right]\left\{\begin{array}{l}
A_{1} \\
A_{2}
\end{array}\right\}
$$

where

$$
\mathbf{\Lambda}_{\mathbf{2 0}}^{\mathbf{R}}=\left[\begin{array}{cc}
-i k_{1} & 0 \\
0 & -i k_{2}
\end{array}\right] \text { and } \boldsymbol{\Lambda}_{\mathbf{2} \mathbf{L}}^{\mathbf{R}}=\left[\begin{array}{cc}
-i k_{1} e^{-i k_{1} L_{x}} & 0 \\
0 & -i k_{2} e^{-i k_{2} L_{x}}
\end{array}\right] .
$$

The expressions in Eqs (20) and (21) can be written in compact matrix form as:

$$
\mathbf{u}=\mathbf{\Psi} \mathbf{A}
$$

and:

$$
\mathbf{F}=\overline{\mathbf{\Psi}} \mathbf{A}
$$

Since $\boldsymbol{\Psi}$ and $\overline{\boldsymbol{\Psi}}$ are $\left(2 m_{x} \times n\right)$ matrices, where $n=2<2 m_{x}$, the systems of equations in Eqs (22) and (23) are overdetermined, and the direct inversion of $\boldsymbol{\Psi}$ is not possible. In order to write the nodal applied forces as a function of the nodal displacements, both sides of Eq. (22) are multiplied by the conjugate transpose of $\boldsymbol{\Psi}$, which allows the computation of the invertible Hermitian matrix $\boldsymbol{\Psi}^{H} \boldsymbol{\Psi}$ and, as a consequence, to write the constant vector $\mathbf{A}$ as a function of the nodal displacements.

$$
\begin{aligned}
& \boldsymbol{\Psi}^{H} \mathbf{u}=\boldsymbol{\Psi}^{H} \boldsymbol{\Psi} \boldsymbol{A} \\
& \mathbf{A}=\left(\boldsymbol{\Psi}^{H} \boldsymbol{\Psi}\right)^{-\mathbf{1}} \boldsymbol{\Psi}^{H} \mathbf{u}
\end{aligned}
$$

Substituting Eq. (25) into Eq. (23), one arrives at the force-displacement relation:

$$
\mathbf{F}=\overline{\boldsymbol{\Psi}}\left(\boldsymbol{\Psi}^{H} \boldsymbol{\Psi}\right)^{-\mathbf{1}} \boldsymbol{\Psi}^{H} \mathbf{u}
$$

where:

$$
\mathbf{K}(\omega)=\bar{\Psi}\left(\Psi^{H} \mathbf{\Psi}\right)^{-\mathbf{1}} \boldsymbol{\Psi}^{H}
$$




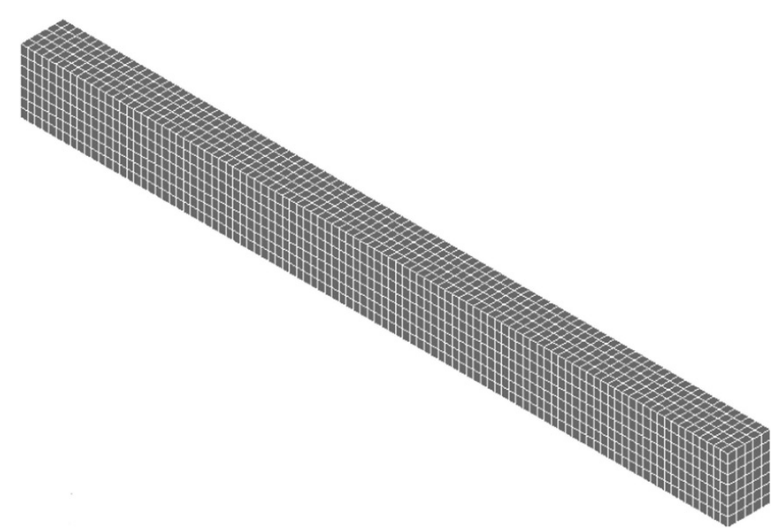

(a)

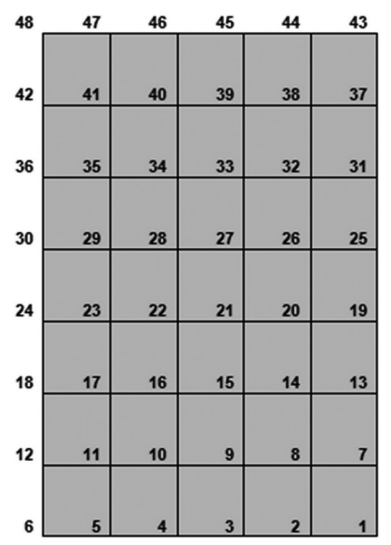

(b)

Fig. 6. (a) Finite Element mesh of the beam used in the numerical examples, (b) Cross section of the beam with numbered nodes.

Equation (27) gives the expression for the numerical spectral element matrix of the rod including all the nodal displacements. Matrix $\mathbf{K}(\omega)$ is square and, if only a few propagation modes are kept compared to the total number of degrees of freedom that discretize the cross section, it is close to singular. Therefore, the solution to Eq. (26) is obtained by a pseudo-inversion using the Singular Value Decomposition (SVD) [13]. Solving Eq. (26) for the longitudinal displacements gives:

$$
\mathbf{u}=\mathbf{K}(\omega)^{+} \mathbf{F}
$$

where $\mathbf{K}(\omega)^{+}$is the pseudo-inverse of a matrix obtained via SVD.

The procedure just described allows the determination of the displacement at each node of the rod cross section. It is important to mention here that, even though only longitudinal deformation was assumed, considering that the wave mode information for each node of the cross section modeled with solid finite elements was considered (instead of obtaining average cross section displacements and forces), the investigation of cross section behavior as frequency rises is possible.

Alternatively, the rod response can be condensed into one node per cross section, as does the elementary theory. This is done by assuming that all cross sectional nodes have the same displacements, which yields:

$$
\left\{\begin{array}{l}
\mathbf{u}_{\mathbf{0}} \\
\mathbf{u}_{\mathbf{L}}
\end{array}\right\}=\left[\begin{array}{cc}
1 & 0 \\
\vdots & \vdots \\
1 & 0 \\
-- & -- \\
0 & 1 \\
\vdots & \vdots \\
0 & 1
\end{array}\right]\left\{\begin{array}{l}
\bar{u}_{0} \\
\bar{u}_{L}
\end{array}\right\}=\mathbf{P}_{2 m_{x} \times 2}\left\{\begin{array}{l}
\bar{u}_{0} \\
\bar{u}_{L}
\end{array}\right\}
$$

Thus, substituting Eq. (22) into Eq. (29) gives:

$$
\left\{\begin{array}{l}
\mathbf{u}_{\mathbf{0}} \\
\mathbf{u}_{\mathbf{L}}
\end{array}\right\}=\mathbf{\Psi} \mathbf{A}=\mathbf{P}_{2 m_{x} \times 2}\left\{\begin{array}{c}
\bar{u}_{0} \\
\bar{u}_{L}
\end{array}\right\}
$$

and vector $\mathbf{A}$ is given by:

$$
\mathbf{A}=\left(\mathbf{P}^{\mathbf{T}} \boldsymbol{\Psi}\right)^{-1} \mathbf{P}^{T} \mathbf{P}\left\{\begin{array}{c}
\bar{u}_{0} \\
\bar{u}_{L}
\end{array}\right\}
$$




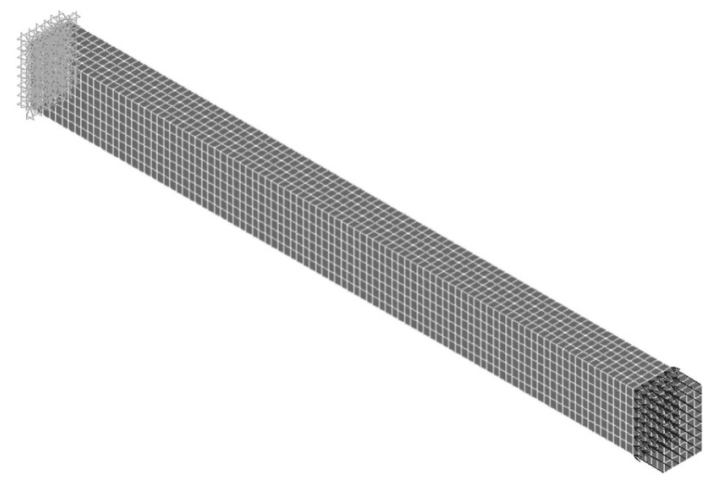

Fig. 7. Finite Element mesh of a cantilever beam subjected to uniform longitudinal force (rod case).

Besides, the resultant force at each side of the rod spectral element can be obtained as the sum of the forces acting at each node of the discretized cross section, which gives:

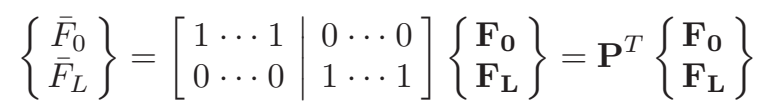

Substituting Eq. (23) into Eq. (32), and, finally, using Eq. (31), yields:

$$
\left\{\begin{array}{l}
\bar{F}_{0} \\
\bar{F}_{L}
\end{array}\right\}=\mathbf{P}^{T} \overline{\boldsymbol{\Psi}}\left(\mathbf{P}^{T} \boldsymbol{\Psi}\right)^{-1} \mathbf{P}^{T} \mathbf{P}\left\{\begin{array}{l}
\bar{u}_{0} \\
\bar{u}_{L}
\end{array}\right\}
$$

The condensed numerical spectral matrix is, thus, given by:

$$
\mathbf{K}_{\mathbf{c}}(\omega)=\mathbf{P}^{T} \bar{\Psi}\left(\mathbf{P}^{T} \mathbf{\Psi}\right)^{-1} \mathbf{P}^{T} \mathbf{P}
$$

\subsection{Forced response for the rod case}

In this sub-section, the WSFEM is applied to compute the forced response of the beam previously described, but with length $L_{x}=100 \mathrm{~mm}$. The finite element model of the full beam was obtained using ANSYS ${ }^{\circledR}$ and has 3,500 SOLID45 elements. The corresponding FE mesh is presented in Fig. 6(a). In Fig. 6(b) the nodes on a cross section are numbered. The SOLID45 element is defined by eight nodes having three degrees of freedom corresponding to translations in the $x, y$, and $z$ directions.

Consider the forced response of a cantilever beam subjected to a unitary axial dynamic force applied to all nodes at the free end of the beam, as presented in Fig. 7. Given the boundary and load conditions, it will be treated as a rod. For this forced response case, only the longitudinal wavenumber pair and its corresponding wave propagation modes were used. The dynamic stiffness matrix was obtained using Eq. (27) when considering all the nodes at the free end, and using Eq. (34) for the condensed DOF case. The results in terms of the longitudinal displacement at the free end of the simulated rod using the condensed and non-condensed formulations are shown in Fig. 8(a).

A good agreement with the result obtained from conventional finite element analysis of the structure performed with ANSYS ${ }^{\circledR}$ is obtained, especially at the resonance peaks. Nevertheless, it is clear that discrepancies occur at the anti-resonances between the responses evaluated by WSFEM and FEM. The response at the anti-resonances is known to be very sensitive to contributions of higher-order modes, which are taken into account in the FE analysis, but not in the WSFEM formulations presented in this paper.

In Fig. 8(b), the relative errors between the numerical and analytical responses are plotted. The greater differences occur between finite element and spectral element results. This can be attributed to the WSFEM formulations being based on the elementary rod theory. The applied forces are written as a function of the displacement field using the elastodynamic equations for the rod, and only longitudinal propagation modes were taken into account. This feature of the WSFEM, associated with the possibility of evaluating the numerical response at each node of the cross section, confirm it as an intermediate numerical approach lying between FEM and standard SEM. 


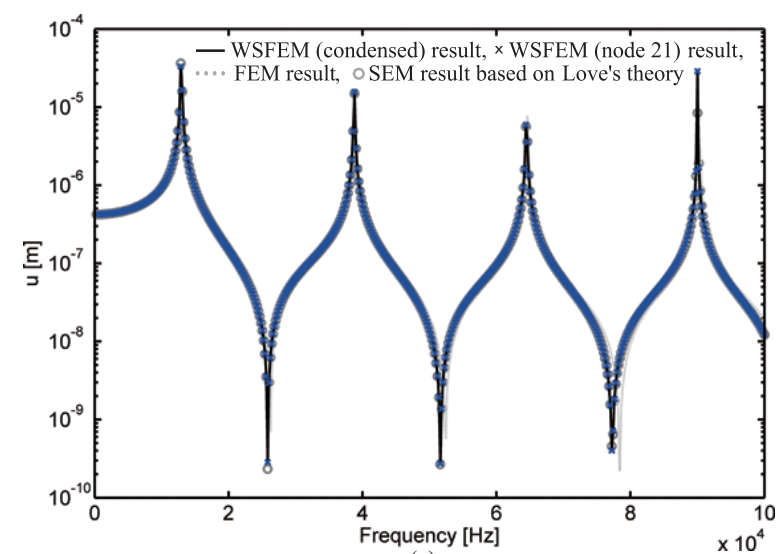

(a)

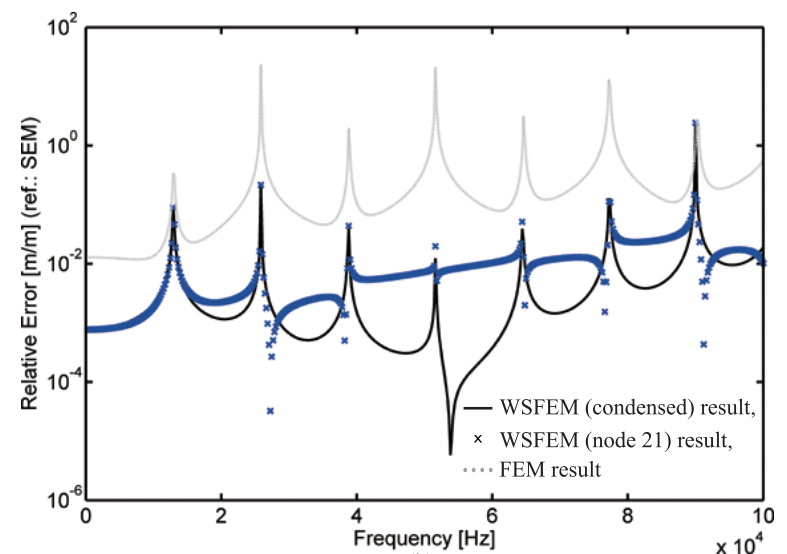

(b)

Fig. 8. (a) Displacement of the rod for a longitudinal uniform force distribution, (b) Relative error between numerical solutions (WSFEM and FEM) and the analytical one (SEM).

\subsection{Derivation of the numerical spectral element matrix for an Euler Bernoulli beam}

In this sub-section, the procedure to obtain the numerical spectral element matrix of an Euler Bernoulli beam using WSFEM is presented. The Euler-Bernoulli beam theory assumes that the deflection of the neutral axis $v(x, t)$ is small and only transverse. It also assumes that the vertical deflection is approximately constant over the cross section and that the horizontal displacement follows the "plane sections remain plane" assumption. Moreover, the presence of a transverse shear force is considered, but the shear deformation due to it is neglected.

The transverse deflection in the $y$ direction for the beam can be expanded as a function of the wavenumbers and wave propagation modes as:

$$
\mathbf{v}=\sum_{r} A_{r} \boldsymbol{\Phi}_{\mathbf{y r}} e^{-i k_{r} x}
$$

where, as before, $A_{r}$ are the undetermined wave amplitudes, $k_{r}$ are the wavenumbers for the bending waves and $\boldsymbol{\Phi}_{\mathbf{y r}}$ are the corresponding wave propagation modes associated with the degrees of freedom in the $y$ direction. These are obtained from the eigenvalue decomposition of the transfer matrix obtained from the finite element model of the beam slice (Fig. 2) as detailed in Section 2.

The rotations about the $z$ axis can be written as:

$$
\boldsymbol{\varphi}=\frac{\partial \mathbf{v}}{\partial x}=\sum_{r}-i k_{r} A_{r} \boldsymbol{\Phi}_{\mathbf{y r}} e^{-i k_{r} x}
$$

Using Eqs (35) and (36), the displacements and rotations at the end nodes of the element can be written in matrix form for an element of arbitrary length $L_{x}$ as:

$$
\left\{\begin{array}{c}
\mathbf{v}_{\mathbf{0}} \\
\varphi_{\mathbf{0}} \\
\mathbf{v}_{\mathbf{L}} \\
\varphi_{\mathbf{L}}
\end{array}\right\}=\left[\begin{array}{c}
\Phi_{\mathbf{y}} \Lambda_{10}^{\mathrm{B}} \\
\boldsymbol{\Phi}_{\mathbf{y}} \Lambda_{20}^{\mathrm{B}} \\
\boldsymbol{\Phi}_{\mathbf{y}} \Lambda_{1 \mathrm{~L}}^{\mathrm{B}} \\
\boldsymbol{\Phi}_{\mathbf{y}} \Lambda_{2 \mathrm{~L}}^{\mathrm{B}}
\end{array}\right]\left\{\begin{array}{c}
A_{1} \\
A_{2} \\
A_{3} \\
A_{4}
\end{array}\right\}
$$

where:

$$
\Phi_{\mathrm{y}}=\left[\begin{array}{llll}
\Phi_{\mathrm{y} 1} & \boldsymbol{\Phi}_{\mathrm{y} 2} & \Phi_{\mathrm{y} 3} & \Phi_{\mathrm{y} 4}
\end{array}\right]
$$




$$
\begin{aligned}
& \mathbf{\Lambda}_{\mathbf{1 0}}^{\mathbf{B}}=\left[\begin{array}{llll}
1 & 0 & 0 & 0 \\
0 & 1 & 0 & 0 \\
0 & 0 & 1 & 0 \\
0 & 0 & 0 & 1
\end{array}\right] \\
& \mathbf{\Lambda}_{\mathbf{1 L}}^{\mathbf{B}}=\left[\begin{array}{cccc}
e^{-i k_{1} L_{x}} & 0 & 0 & 0 \\
0 & e^{-i k_{2} L_{x}} & 0 & 0 \\
0 & 0 & e^{-i k_{3} L_{x}} & 0 \\
0 & 0 & 0 & e^{-i k_{4} L_{x}}
\end{array}\right] \\
& \boldsymbol{\Lambda}_{\mathbf{2 0}}^{\mathbf{B}}=\left[\begin{array}{cccc}
-i k_{1} & 0 & 0 & 0 \\
0 & -i k_{2} & 0 & 0 \\
0 & 0 & -i k_{3} & 0 \\
0 & 0 & 0 & -i k_{4}
\end{array}\right] \\
& \mathbf{\Lambda}_{\mathbf{2} \mathbf{L}}^{\mathbf{B}}=\left[\begin{array}{cccc}
-i k_{1} e^{-i k_{1} L_{x}} & 0 & 0 & 0 \\
0 & -i k_{2} e^{-i k_{2} L_{x}} & 0 & 0 \\
0 & 0 & -i k_{3} e^{-i k_{3} L_{x}} & 0 \\
0 & 0 & 0 & -i k_{4} e^{-i k_{4} L_{x}}
\end{array}\right]
\end{aligned}
$$

It is assumed here that $r=1, \ldots, 4$ correspond to the bending and shear around the $z$-axis wavenumbers and wave propagation modes.

An Euler-Bernoulli beam element is subjected to the following internal bending moment and transverse shear force, respectively:

$$
\begin{aligned}
& V=-E I \frac{\partial^{3} v}{\partial x^{3}} \\
& M=E I \frac{\partial^{2} v}{\partial x^{2}}
\end{aligned}
$$

where $I$ is the second moment of inertia w.r.t. the $z$-axis.

Substituting Eq. (35) into Eqs (39) and (40) yields the following spectral solutions for the applied force and moment:

$$
\begin{aligned}
& \mathbf{V}=-E \mathbf{I}_{\mathbf{e}} \sum_{r}\left(i k_{r}^{3}\right) A_{r} \boldsymbol{\Phi}_{\mathbf{y r}} e^{-i k_{r} x} \\
& \mathbf{M}=E \mathbf{I}_{\mathbf{e}} \sum_{r}\left(-k_{r}^{2}\right) A_{r} \boldsymbol{\Phi}_{\mathbf{y r}} e^{-i k_{r} x}
\end{aligned}
$$

where $\mathbf{I}_{\mathbf{e}}$ is the diagonal matrix that provides the moments of inertia associated with each elemental area surrounding a given node, as follows:

$$
I_{e}=\int y^{2} d S \approx I_{e}^{0}+y^{2} S_{e}
$$

Equations (41) and (42) can be rewritten as:

$$
\left\{\begin{array}{c}
\mathbf{V}_{\mathbf{0}} \\
\mathbf{M}_{\mathbf{0}} \\
\mathbf{V}_{\mathbf{L}} \\
\mathbf{M}_{\mathbf{L}}
\end{array}\right\}=E\left[\begin{array}{c}
-\mathbf{I}_{\mathbf{e}} \Phi_{\mathbf{y}} \Lambda_{30}^{\mathrm{B}} \\
\mathbf{I}_{\mathbf{e}} \Phi_{\mathbf{y}} \Lambda_{40}^{\mathrm{B}} \\
\mathbf{I}_{\mathbf{e}} \Phi_{\mathbf{y}} \Lambda_{3 \mathbf{L}}^{\mathrm{B}} \\
-\mathbf{I}_{\mathbf{e}} \Phi_{\mathbf{y}} \Lambda_{4 \mathbf{L}}^{\mathrm{B}}
\end{array}\right]\left\{\begin{array}{l}
A_{1} \\
A_{2} \\
A_{3} \\
A_{4}
\end{array}\right\}
$$

and

$$
\mathbf{\Lambda}_{\mathbf{3 0}}^{\mathbf{B}}=\left[\begin{array}{cccc}
i k_{1}^{3} & 0 & 0 & 0 \\
0 & i k_{2}^{3} & 0 & 0 \\
0 & 0 & i k_{3}^{3} & 0 \\
0 & 0 & 0 & i k_{4}^{3}
\end{array}\right]
$$




$$
\begin{aligned}
\boldsymbol{\Lambda}_{\mathbf{3} \mathbf{L}}^{\mathbf{B}} & =\left[\begin{array}{cccc}
i k_{1}^{3} e^{-i k_{1} L} & 0 & 0 & 0 \\
0 & i k_{2}^{3} e^{-i k_{2} L} & 0 & 0 \\
0 & 0 & i k_{3}^{3-i k_{3} L} & 0 \\
0 & 0 & 0 & i k_{4}^{3} e^{-i k_{4} L}
\end{array}\right] \\
\boldsymbol{\Lambda}_{\mathbf{4 0}}^{\mathbf{B}} & =\left[\begin{array}{cccc}
-k_{1}^{2} & 0 & 0 & 0 \\
0 & -k_{2}^{2} & 0 & 0 \\
0 & 0 & -k_{3}^{2} & 0 \\
0 & 0 & 0 & -k_{4}^{2}
\end{array}\right] \\
\boldsymbol{\Lambda}_{\mathbf{4} \mathbf{L}}^{\mathbf{B}} & =\left[\begin{array}{cccc}
-k_{1}^{2} e^{-i k_{1} L} & 0 & 0 & 0 \\
0 & -k_{2}^{2} e^{-i k_{2} L} & 0 & 0 \\
0 & 0 & -k_{3}^{2} e^{-i k_{3} L} & 0 \\
0 & 0 & 0 & -k_{4}^{2} e^{-i k_{4} L}
\end{array}\right]
\end{aligned}
$$

The expressions in Eqs (37) and (44) can be written in compact matrix form as:

$$
\left\{\begin{array}{l}
\mathrm{v} \\
\varphi
\end{array}\right\}=\Psi_{\mathrm{b}} \mathrm{A}
$$

and:

$$
\left\{\begin{array}{c}
\mathbf{F} \\
\mathbf{M}
\end{array}\right\}=\overline{\mathbf{\Psi}}_{\mathbf{b}} \mathbf{A}
$$

where:

$$
\begin{aligned}
& \Psi_{\mathrm{b}}=\left[\begin{array}{l}
\Phi_{\mathrm{y}} \Lambda_{10}^{\mathrm{B}} \\
\Phi_{\mathrm{y}} \Lambda_{20}^{\mathrm{B}} \\
\Phi_{\mathrm{y}} \Lambda_{1 \mathrm{~L}}^{\mathrm{B}} \\
\Phi_{\mathrm{y}} \Lambda_{2 \mathrm{~L}}^{\mathrm{B}}
\end{array}\right] \\
& \overline{\mathbf{\Psi}}_{\mathrm{b}}=E\left[\begin{array}{c}
-\mathbf{I}_{\mathrm{e}} \Phi_{\mathrm{y}} \Lambda_{30}^{\mathrm{B}} \\
\mathbf{I}_{\mathbf{e}} \Phi_{\mathrm{y}} \Lambda_{40}^{\mathrm{B}} \\
\mathbf{I}_{\mathrm{e}} \Phi_{\mathrm{y}} \Lambda_{3 \mathrm{~L}}^{\mathrm{B}} \\
-\mathbf{I}_{\mathrm{e}} \Phi_{\mathrm{y}} \Lambda_{4 \mathrm{~L}}^{\mathrm{B}}
\end{array}\right]
\end{aligned}
$$

Differently from the rod case, in the beam case, the displacement field is described by two dependent variables: the transverse displacement and the rotation about the $z$-axis. In this case only the condensed numerical spectral element matrix was obtained, which is consistent with the theory applied (Euler-Bernoulli). Repeating the procedure explained in Sub-Section 3.1 from Eq. (29) to Eq. (34), this matrix is obtained for the new matrices $\boldsymbol{\Psi}_{\mathbf{b}}$ and $\bar{\Psi}_{\mathbf{b}}$ that have just been derived for an Euler-Bernoulli beam. So, after further manipulation, one can arrive at:

$$
\left\{\begin{array}{c}
\mathbf{F} \\
\mathbf{M}
\end{array}\right\}=\mathbf{P}^{T} \overline{\mathbf{\Psi}}_{\mathbf{b}}\left(\mathbf{P}^{T} \boldsymbol{\Psi}_{\mathbf{b}}\right)^{-1} \mathbf{P}^{T} \mathbf{P}\left\{\begin{array}{c}
\mathbf{v} \\
\boldsymbol{\varphi}
\end{array}\right\}
$$

where:

$$
\mathbf{K}_{\mathbf{c b}}(\omega)=\mathbf{P}^{T} \overline{\mathbf{\Psi}}_{\mathbf{b}}\left(\mathbf{P}^{T} \boldsymbol{\Psi}_{\mathbf{b}}\right)^{-1} \mathbf{P}^{T} \mathbf{P}
$$

Solving Eq. (49) for the transverse displacements and rotations gives:

$$
\left\{\begin{array}{l}
\mathbf{v} \\
\varphi
\end{array}\right\}=\mathbf{K}_{\mathbf{c b}}(\omega)^{+}\left\{\begin{array}{c}
\mathbf{F} \\
\mathbf{M}
\end{array}\right\}
$$

where $\mathbf{K}_{\mathbf{c b}}(\omega)^{+}$is the pseudo-inverse of $\mathbf{K}_{\mathbf{c b}}(\omega)$. 


\subsection{Derivation of the numerical spectral element matrix for a Timoshenko beam}

In this sub-section, the Timoshenko beam theory is used in order to derive the numerical spectral matrix of a beam. The Timoshenko formulation takes into account the shear deformation and the rotational inertia. It is more suitable for describing the behavior of short beams or high frequency effects. The degrees of freedom considered in this formulation are the transverse displacements and rotations. From the Timoshenko theory, the rotation about the $z$-axis is given by:

$$
\varphi=-\frac{\partial u}{\partial y}
$$

Using the governing differential equations of a Timoshenko beam [9], the expression above can also be written as:

$$
\frac{\partial \varphi}{\partial x}=\left(\kappa G \frac{\partial^{2} v}{\partial x^{2}}-\rho \omega^{2} v\right) \frac{1}{\kappa G}, \quad \varphi=\left(\kappa G k^{2}(\omega)+\rho \omega^{2}\right) \frac{v}{i \kappa G k(\omega)}
$$

where $G=E / 2(1+\nu)$ is the shear modulus and $\kappa$ is the shear coefficient $(\kappa=0.89$ was adopted in this paper for the simulated beam with rectangular cross section).

As before, the longitudinal and transverse displacements can be expanded in a sum of wave propagation modes.

$$
\begin{aligned}
& \mathbf{u}=\sum_{r} A_{r} \boldsymbol{\Phi}_{\mathbf{x r}} e^{-i k_{r} x} \\
& \mathbf{v}=\sum_{r} A_{r} \boldsymbol{\Phi}_{\mathbf{y r}} e^{-i k_{r} x}
\end{aligned}
$$

where $k_{r}$ are the wavenumbers and $\boldsymbol{\Phi}_{\mathbf{x r}}$ and $\boldsymbol{\Phi}_{\mathbf{y r}}$ are the wave propagation modes associated with $x$ and $y$ degrees of freedom for the bending and shear propagation modes. These were obtained from the eigenvalue decomposition of the transfer matrix obtained from the finite element model of the beam slice (Fig. 2), as detailed in Section 2.

Substituting Eqs (54) and (55) into Eqs (52) or (53), the rotation can be written in spectral form:

$$
\begin{aligned}
& \boldsymbol{\varphi}=-\sum_{r} A_{r} \frac{\partial \boldsymbol{\Phi}_{\mathbf{x r}}}{\partial y} e^{-i k_{r} x} \\
& \boldsymbol{\varphi}=\sum_{r} A_{r} \frac{\left(\kappa G k_{r}^{2}+\rho \omega^{2}\right)}{i \kappa G k_{r}} \boldsymbol{\Phi}_{\mathbf{y r}} e^{-i k_{r} x}
\end{aligned}
$$

The first expression, Eq. (56), requires the derivative of the eigenvector component related to the displacement in the $x$ direction for each node of the cross section. Finite differences can be used in order to compute this derivative.

The expressions for the forces applied at the nodes of a Timoshenko beam element can be expanded in a sum of wave propagation modes. The transverse or shear force is obtained by integration of the shear stress:

$$
V=f_{y}=-\kappa \int G \gamma_{x y} d S=-\kappa G S\left(\frac{\partial u}{\partial y}+\frac{\partial v}{\partial x}\right)=-\kappa G S\left(\frac{\partial v}{\partial x}-\varphi\right)
$$

Substituting Eqs (54) and (55) into Eq. (58), the shear force can be written as:

$$
\mathbf{V}=-\kappa G S_{e}\left(\sum_{r} A_{r} \frac{\partial \boldsymbol{\Phi}_{\mathbf{x r}}}{\partial y} e^{-i k_{r} x}+\sum_{r}-i k_{r} A_{r} \boldsymbol{\Phi}_{\mathbf{y r}} e^{-i k_{r} x}\right)
$$

or, using Eq. (57), as:

$$
\mathbf{V}=-\kappa G S_{e}\left(\sum_{r}-i k_{r} A_{r} \boldsymbol{\Phi}_{\mathbf{y r}} e^{-i k_{r} x}-\sum_{r} A_{r} \frac{\left(\kappa G k_{r}^{2}+\rho \omega^{2}\right)}{i \kappa G k_{r}} \boldsymbol{\Phi}_{\mathbf{y r}} e^{-i k_{r} x}\right)
$$


And the moment about the $z$-axis is given by:

$$
M=m_{z}=E I_{z} \frac{\partial \varphi}{\partial x}
$$

In the spectral form, this can be written as:

$$
\mathbf{M}=-E I_{z} \sum_{r}-i k_{r} A_{r} \frac{\partial \mathbf{\Phi}_{\mathbf{x r}}}{\partial y} e^{-i k_{r} x}
$$

or

$$
\mathbf{M}=E I_{z} \sum_{r}-i k_{r} A_{r} \frac{\left(\kappa G k_{r}^{2}+\rho \omega^{2}\right)}{i \kappa G k_{r}} \mathbf{\Phi}_{\mathbf{y r}} e^{-i k_{r} x}
$$

Using Eqs (55) and (57), the transverse displacements and rotations at the end nodes of the element can be written as a function of the wavenumbers and corresponding wave propagation modes in matrix form for an element of arbitrary length $L_{x}$ as:

$$
\left\{\begin{array}{c}
\mathbf{v}_{\mathbf{0}} \\
\varphi_{\mathbf{0}} \\
\mathbf{v}_{\mathbf{L}} \\
\varphi_{\mathbf{L}}
\end{array}\right\}=\left[\begin{array}{c}
\boldsymbol{\Phi}_{\mathbf{y}} \boldsymbol{\Lambda}_{10}^{\mathrm{B}} \\
\boldsymbol{\Phi}_{\mathbf{y}} \boldsymbol{\Lambda}_{50}^{\mathrm{B}} \\
\boldsymbol{\Phi}_{\mathbf{y}} \boldsymbol{\Lambda}_{1 \mathrm{~L}}^{\mathrm{B}} \\
\boldsymbol{\Phi}_{\mathbf{y}} \boldsymbol{\Lambda}_{5 \mathbf{L}}^{\mathrm{B}}
\end{array}\right]\left\{\begin{array}{l}
A_{1} \\
A_{2} \\
A_{3} \\
A_{4}
\end{array}\right\}
$$

where $\Lambda_{10}^{\mathrm{B}}, \Lambda_{1 \mathrm{~L}}^{\mathrm{B}}$ are given by matrices in Eqs (38.2) and (38.3) and

$$
\begin{aligned}
\boldsymbol{\Lambda}_{\mathbf{5 0}}^{\mathbf{B}} & =\left[\begin{array}{cccc}
D_{1} & 0 & 0 & 0 \\
0 & D_{2} & 0 & 0 \\
0 & 0 & D_{3} & 0 \\
0 & 0 & 0 & D_{4}
\end{array}\right] \\
\boldsymbol{\Lambda}_{\mathbf{5} \mathbf{L}}^{\mathbf{B}} & =\left[\begin{array}{cccc}
D_{1} e^{-i k_{1} L_{x}} & 0 & 0 & 0 \\
0 & D_{2} e^{-i k_{2} L_{x}} & 0 & 0 \\
0 & 0 & D_{3} e^{-i k_{3} L_{x}} & 0 \\
0 & 0 & 0 & D_{4} e^{-i k_{4} L_{x}}
\end{array}\right]
\end{aligned}
$$

where $D_{r}=\left(\kappa G k_{r}^{2}+\rho \omega^{2}\right) / i \kappa G k_{r}, r=1, \ldots, 4$.

In the same way, using Eqs (60) and (63), the applied forces and moments at $x=0$ and $x=L_{x}$ can be written in matrix form as:

$$
\left\{\begin{array}{c}
\mathbf{V}_{\mathbf{0}} \\
\mathbf{M}_{\mathbf{0}} \\
\mathbf{V}_{\mathbf{L}} \\
\mathbf{M}_{\mathbf{L}}
\end{array}\right\}=\left[\begin{array}{c}
-\kappa G A_{e} \boldsymbol{\Phi}_{\mathbf{y}}\left(\boldsymbol{\Lambda}_{\mathbf{2 0}}^{\mathrm{B}}-\boldsymbol{\Lambda}_{\mathbf{5 0}}^{\mathrm{B}}\right) \\
E \mathbf{I}_{\mathbf{e}} \mathbf{\Phi}_{\mathbf{y}} \boldsymbol{\Lambda}_{6 \mathbf{6}}^{\mathrm{B}} \\
\kappa G A_{e} \mathbf{\Phi}_{\mathbf{y}}\left(\boldsymbol{\Lambda}_{\mathbf{2}}^{\mathrm{B}}-\boldsymbol{\Lambda}_{\mathbf{5}}^{\mathrm{B}}\right) \\
-E \mathbf{I}_{\mathbf{e}} \mathbf{\Phi}_{\mathbf{y}} \boldsymbol{\Lambda}_{\mathbf{6} \mathbf{L}}^{\mathrm{B}}
\end{array}\right]\left\{\begin{array}{c}
A_{1} \\
A_{2} \\
A_{3} \\
A_{4}
\end{array}\right\}
$$

where

$$
\boldsymbol{\Lambda}_{\mathbf{6 0}}^{\mathbf{B}}=\left[\begin{array}{cccc}
-i k_{1} D_{1} & 0 & 0 & 0 \\
0 & -i k_{2} D_{2} & 0 & 0 \\
0 & 0 & -i k_{3} D_{3} & 0 \\
0 & 0 & 0 & -i k_{4} D_{4}
\end{array}\right]
$$




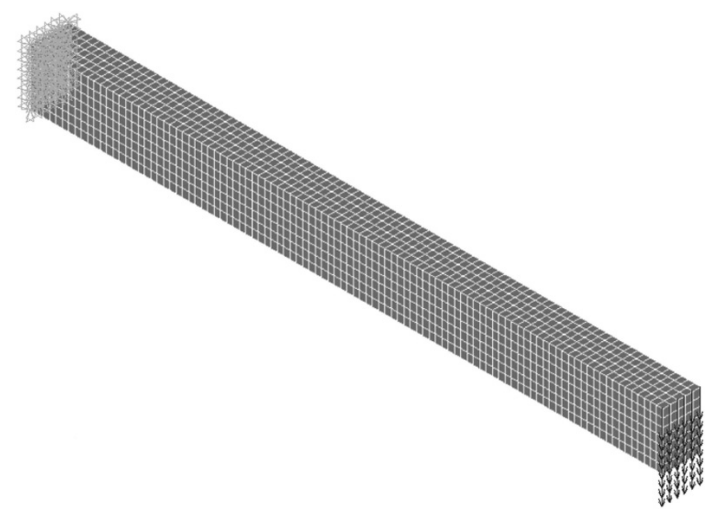

Fig. 9. Finite Element mesh of cantilever beam subjected to a transverse force (beam case).

$$
\mathbf{\Lambda}_{\mathbf{6} \mathbf{L}}^{\mathbf{B}}=\left[\begin{array}{cccc}
-i k_{1} D_{1} e^{-i k_{1} L_{x}} & 0 & 0 & 0 \\
0 & -i k_{2} D_{2} e^{-i k_{2} L_{x}} & 0 & 0 \\
0 & 0 & -i k_{3} D_{3} e^{-i k_{3} L_{x}} & 0 \\
0 & 0 & 0 & -i k_{4} D_{4} e^{-i k_{4} L_{x}}
\end{array}\right]
$$

The matrix expressions in Eqs (64) and (66) can be written in compact matrix form as:

$$
\begin{aligned}
& \left\{\begin{array}{l}
\mathrm{v} \\
\varphi
\end{array}\right\}=\Psi_{\mathrm{T}} \mathrm{A} \\
& \left\{\begin{array}{c}
\mathrm{V} \\
\mathrm{M}
\end{array}\right\}=\overline{\mathbf{\Psi}}_{\mathbf{T}} \mathrm{A}
\end{aligned}
$$

As in the Euler-Bernoulli case, only the condensed numerical spectral element matrix was obtained using this approach for a Timoshenko beam. Thus, repeating the procedure explained in Sub-Section 3.1 from Eq. (29) to Eq. (34), this matrix is obtained for the new matrices $\boldsymbol{\Psi}_{\mathbf{T}}$ and $\bar{\Psi}_{\mathbf{T}}$ that have just been derived for the Timoshenko beam. So, after further manipulation, one arrives at:

$$
\left\{\begin{array}{c}
\mathbf{F} \\
\mathbf{M}
\end{array}\right\}=\mathbf{P}^{T} \overline{\mathbf{\Psi}}_{\mathbf{T}}\left(\mathbf{P}^{T} \boldsymbol{\Psi}_{\mathbf{T}}\right)^{-1} \mathbf{P}^{T} \mathbf{P}\left\{\begin{array}{l}
\mathbf{v} \\
\boldsymbol{\varphi}
\end{array}\right\}
$$

where

$$
\mathbf{K}_{\mathbf{c T}}(\omega)=\mathbf{P}^{T} \overline{\mathbf{\Psi}}_{\mathbf{T}}\left(\mathbf{P}^{T} \boldsymbol{\Psi}_{\mathbf{T}}\right)^{-1} \mathbf{P}^{T} \mathbf{P}
$$

is the condensed numerical spectral element matrix for a Timoshenko beam.

Equation (71) gives the condensed numerical spectral element matrix of the beam. Solving Eq. (70) for the transverse displacement and rotation gives:

$$
\left\{\begin{array}{c}
\mathbf{v} \\
\boldsymbol{\varphi}
\end{array}\right\}=\mathbf{K}_{\mathbf{c T}}(\omega)+\left\{\begin{array}{c}
\mathbf{F} \\
\mathbf{M}
\end{array}\right\}
$$

where $\mathbf{K}_{\mathbf{c T}}(\omega)^{+}$is the pseudo-inverse of the dynamic stiffness matrix obtained via SVD.

\subsection{Forced response for the beam case}

In this sub-section, a unitary transverse force is acting at all the nodes at the free end of the beam, as shown in the finite element mesh presented in Fig. 9. The condensed displacement response computed at the free end with Eq. (51) based on the Euler Bernoulli beam theory is shown in Fig. 10(a). The error relative to the full FE 


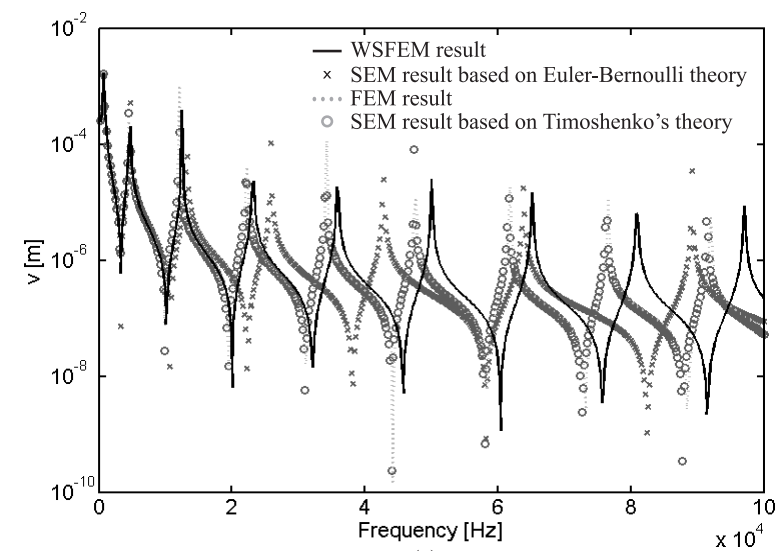

(a)

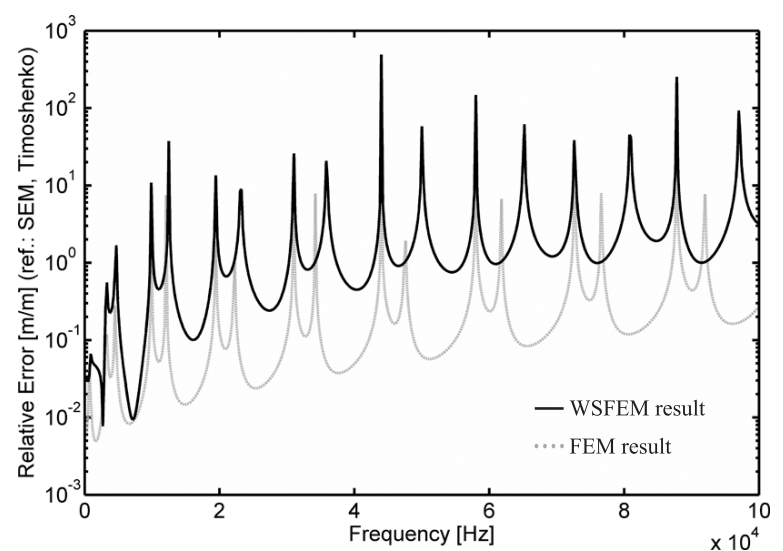

(b)

Fig. 10. (a) Transverse displacement of the beam in the $y$ direction using Euler-Bernoulli theory, (b) relative error between numerical solutions (WSFEM and FEM) and the analytical one (SEM based on Timoshenko's theory).
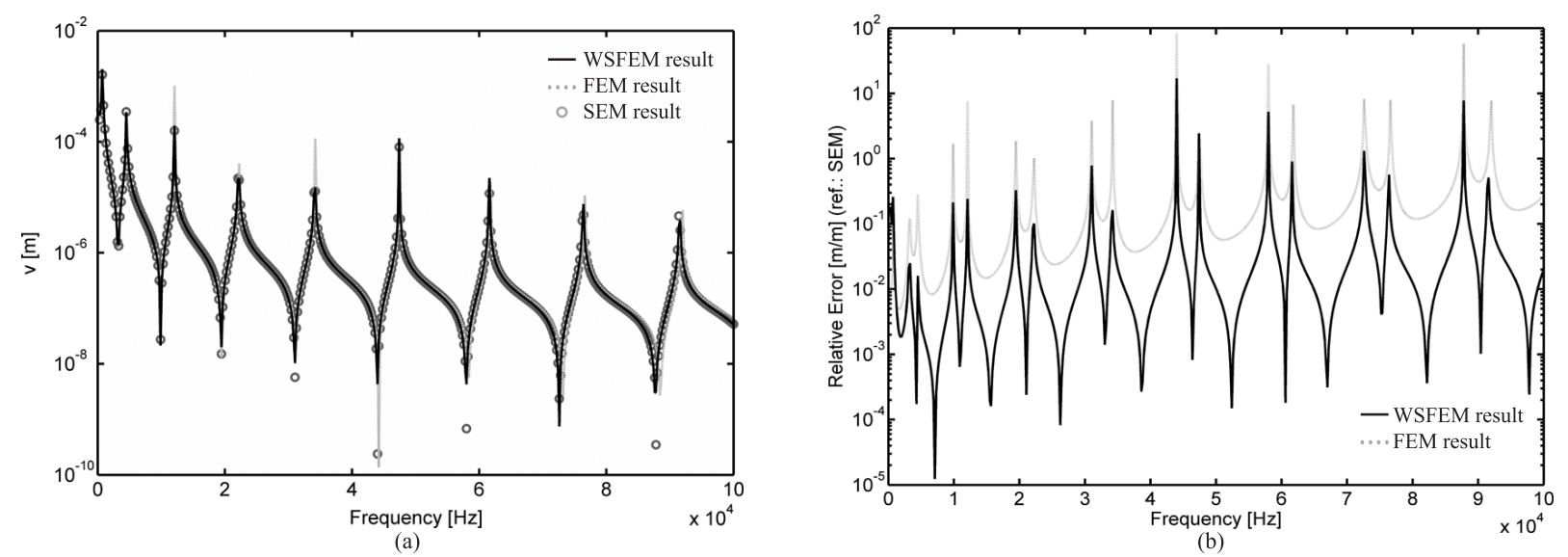

Fig. 11. (a) Transverse displacement of the beam in the $y$ direction using Timoshenko's theory, (b) relative error between numerical solutions (WSFEM and FEM) and the analytical one (SEM).

analysis is plotted in Fig. 10(b). The displacement was also computed using analytical solutions based on EulerBernoulli and Timoshenko beam theories. The comparison shows that a good agreement is obtained only in the low frequency range. It should be pointed out that, although the Euler-Bernoulli governing equations were used to formulate the WSFEM approach in Sub-Section 3.3, in this case the WSFEM is more closely related to the Timoshenko-based standard spectral solution than to the Euler-Bernoulli standard spectral solution. This happens because the wavenumbers predicted by the Euler-Bernoulli beam theory are only valid for low frequencies. In addition, the numerical approach proposed in this paper uses the numerical wavenumbers derived from the FE model of structure slice and, thus, follows the analytical wavenumbers based on the Timoshenko beam theory (see Figs 3 and 4).

The same problem was solved using the WSFEM formulation based on the Timoshenko beam theory (SubSection 3.4). Figures 11(a) and (b) present the results for the condensed transverse displacement computed at the free end using the proposed approach and the associated error relative to the analytical Timoshenko solution, respectively. When compared to the results obtained with a full ANSYS ${ }^{\circledR}$ model of the beam and the analytical Timoshenko results, a good agreement is obtained for most of the frequency range. 


\subsection{Derivation of the numerical spectral element matrix of a beam under off-centered longitudinal load}

In this sub-section, it is assumed that the beam is subjected to an off-centered longitudinal force, which results in displacements in both $x$ and $y$ directions. From expressions in Eqs (54) and (55), the longitudinal displacement in the $x$ direction and the transverse deflection in the $y$ direction can be written as functions of the wavenumbers and wave propagation modes. Then, from Eqs (36) and (57), the rotations are derived using the Euler-Bernoulli and Timoshenko beam theories, respectively. In this case, the spectral element matrix must relate longitudinal displacements and rotations about the $z$-axis with the corresponding applied force and bending moment. Using expressions in Eqs (54) and (57) derived in the previous sub-sections, the displacement field for an element of Timoshenko beam with arbitrary length $L_{x}$ can be written in matrix form as:

$$
\left\{\begin{array}{c}
\mathbf{u}_{\mathbf{0}} \\
\varphi_{\mathbf{0}} \\
\mathbf{u}_{\mathbf{L}} \\
\boldsymbol{\varphi}_{\mathbf{L}}
\end{array}\right\}=\left[\begin{array}{c}
\Phi_{\mathbf{x}} \Lambda_{10}^{\mathrm{B}} \\
\boldsymbol{\Phi}_{\mathbf{y}} \Lambda_{50}^{\mathrm{B}} \\
\boldsymbol{\Phi}_{\mathbf{x}} \Lambda_{1 \mathrm{~L}}^{\mathrm{B}} \\
\boldsymbol{\Phi}_{\mathbf{y}} \Lambda_{5 \mathrm{~L}}^{\mathrm{B}}
\end{array}\right]\left\{\begin{array}{l}
A_{1} \\
A_{2} \\
A_{3} \\
A_{4}
\end{array}\right\}
$$

In this case, the wavenumbers for the longitudinal and bending waves and the corresponding modes associated with $x$ and $y$ degrees of freedom are used. This matrix can be derived in the same way for an Euler-Bernoulli beam subjected to an off-centered axial load.

Using Eqs (19) and (63), the forces and moments at $x=0$ and $x=L_{x}$ can be written in matrix form as:

$$
\left\{\begin{array}{c}
\mathbf{F}_{\mathbf{0}} \\
\mathbf{M}_{\mathbf{0}} \\
\mathbf{F}_{\mathbf{L}} \\
\mathbf{M}_{\mathbf{L}}
\end{array}\right\}=E\left[\begin{array}{c}
-A_{e} \boldsymbol{\Phi}_{\mathbf{x}} \boldsymbol{\Lambda}_{\mathbf{2 0}}^{\mathrm{B}} \\
\mathbf{I}_{\mathbf{e}} \boldsymbol{\Phi}_{\mathbf{y}} \boldsymbol{\Lambda}_{\mathbf{6 0}}^{\mathrm{B}} \\
A_{e} \boldsymbol{\Phi}_{\mathbf{x}} \boldsymbol{\Lambda}_{\mathbf{2}}^{\mathrm{B}} \\
-\mathbf{I}_{\mathbf{e}} \boldsymbol{\Phi}_{\mathbf{y}} \boldsymbol{\Lambda}_{\mathbf{6 L}}^{\mathrm{B}}
\end{array}\right]\left\{\begin{array}{l}
A_{1} \\
A_{2} \\
A_{3} \\
A_{4}
\end{array}\right\}
$$

The expressions in Eqs (73) and (74) can be written in compact matrix form as:

$$
\begin{aligned}
& \left\{\begin{array}{c}
\mathrm{u} \\
\varphi
\end{array}\right\}=\Psi_{\mathrm{O}} \mathrm{A} \\
& \left\{\begin{array}{c}
\mathrm{F} \\
\mathrm{M}
\end{array}\right\}=\bar{\Psi}_{\mathrm{O}} \mathrm{A}
\end{aligned}
$$

Isolating the vector of amplitude constants $\mathbf{A}$ in Eq. (75) and substituting it in Eq. (76) gives:

$$
\left\{\begin{array}{c}
\mathbf{F} \\
\mathbf{M}
\end{array}\right\}=\overline{\boldsymbol{\Psi}}_{\mathbf{O}}\left(\boldsymbol{\Psi}_{\mathbf{O}}^{H} \boldsymbol{\Psi}_{\mathbf{O}}\right)^{-1} \boldsymbol{\Psi}_{\mathbf{O}}^{H}\left\{\begin{array}{l}
\mathbf{u} \\
\boldsymbol{\varphi}
\end{array}\right\}
$$

where:

$$
\mathbf{K}_{\mathbf{O}}(\omega)=\bar{\Psi}_{\mathbf{O}}\left(\Psi_{\mathbf{O}}^{H} \Psi_{\mathbf{O}}\right)^{-1} \Psi_{\mathbf{O}}^{H}
$$

Equation (78) gives the numerical spectral element matrix of the beam including all the nodal displacements. Solving Eq. (77) for the longitudinal displacements and rotations gives:

$$
\left\{\begin{array}{l}
\mathbf{u} \\
\boldsymbol{\varphi}
\end{array}\right\}=\mathbf{K}_{\mathbf{O}}(\omega)^{+}\left\{\begin{array}{c}
\mathbf{F} \\
\mathbf{M}
\end{array}\right\}
$$

where $\mathbf{K}_{\mathbf{O}}(\omega)^{+}$is the pseudo-inverse of the matrix obtained via SVD. 


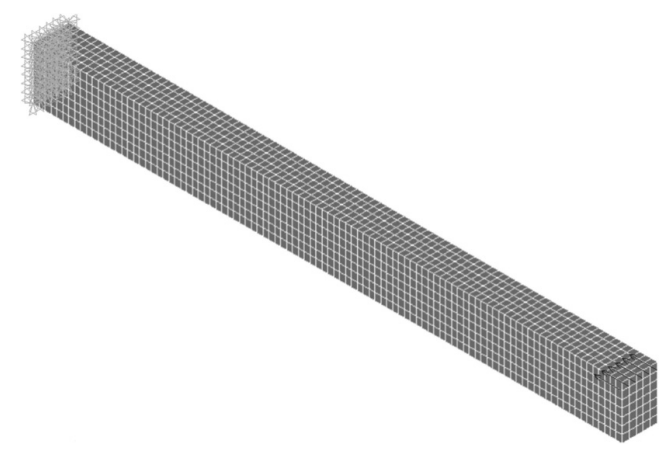

Fig. 12. Element mesh of cantilever beam subjected to a line force in the $x$ direction.

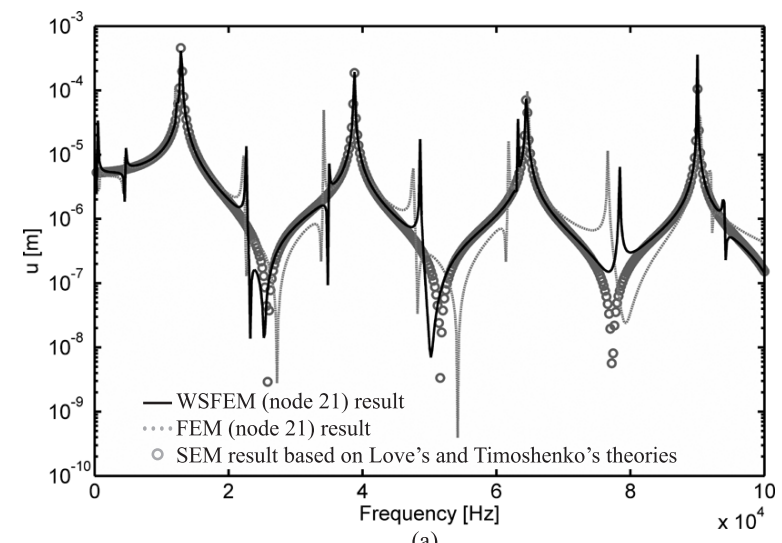

(a)

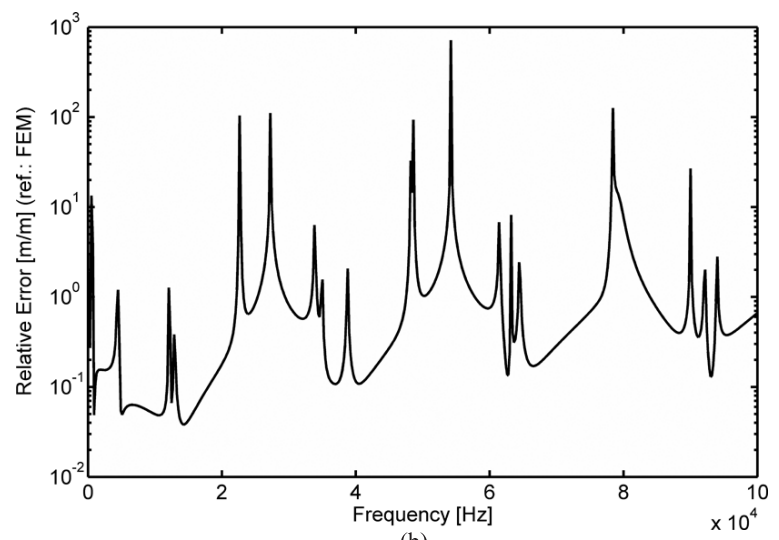

(b)

Fig. 13. (a) Longitudinal displacement of the rod for a non-uniform force distribution, (b) relative error between numerical solutions (WSFEM and FEM).

\subsection{Forced response of a cantilever beam under non-uniform longitudinal load}

Finally, consider the forced response of the beam due to a non-uniform longitudinal line force across the width of the free end surface, $F_{x}=-100 \mathrm{~N}$ applied to each node on the line, as shown in the finite element mesh presented in Fig. 12. Such force distribution will produce both longitudinal and transverse displacements.

The wavenumbers corresponding to longitudinal and bending wave propagation modes are used in the calculations. The computed longitudinal displacement at node 21 at the free end using Eq. (79) presented in Sub-Section 3.6 is shown in Fig. 13(a) compared to the result obtained performing a harmonic response analysis in ANSYS ${ }^{\circledR}$ and the analytical solution for an equivalent force and moment applied to the one-dimensional standard spectral beam element. The relative error between WSFEM and conventional FE analysis is also plotted in Fig. 13(b).

Off-centered loads induce local effects that cannot be simulated when using one-dimensional formulations. From Fig. 13(a), it can be inferred that, although disparities are observed between solutions via WSFEM and FEM, the proposed approach provides, to some extent, information about local effects, while a one-dimensional approach, for instance, standard SEM, which is plotted together in Fig. 13(a), does not provide any local information. This shows the potential of WSFEM as an alternative to computationally expensive conventional FE analyses in midfrequencies.

\section{Conclusion}

In this work it was shown that it is possible to create numerical spectral element matrices of waveguides using the wavenumbers and wave propagation modes obtained from the eigenvalue analysis of the transfer matrix of a 
slice modeled with conventional finite element models. This approach is called here the Wave Spectral Finite Element Method (WSFEM). Initially, a slice of a structural beam was modeled using solid elements and the mass and stiffness matrices were used to derive the transfer matrix. Then, the dispersion curves were obtained from the eigenvalues of the transfer matrix. The numerical dispersion curves were shown to agree well with the analytical ones. Based on elementary theories for rods and beams and using the numerical wavenumbers and wave propagation modes, numerical spectral element matrices of the waveguides were derived. The numerical spectral elements were used to compute the forced response of the beam under clamped-free boundary conditions and subjected to different load conditions, including longitudinal uniform forces, transverse forces and non-uniform longitudinal forces. Results obtained with the WSFEM method were validated by comparison to the results obtained from a conventional finite element analysis and also to analytical solutions provided by the standard spectral element method, showing good agreement. The validation of the results for the beam suggests that it is possible to use this method to obtain numerical spectral element matrices for waveguides with more complex cross sections (geometry and material properties).

The high computational cost of conventional FE analysis of long structural waveguides for mid-frequency problems is the main motivation of this work. Since different commercial software packages have been used to perform FE and WSFE analyses, namely ANSYS ${ }^{\circledR}$ in the first case and MATLAB ${ }^{\circledR}$ in the second case, it is not meaningful to compare the computational cost by comparing the computational times. However, the computational effort can be indirectly compared via the size of the matrices that must be assembled to solve the harmonic problem, dependent on the number of DOF. The FE analyses performed in this paper used a model with 3,500 elements and a total of 14,544 DOF. On the other hand, the WSFEM uses an FE model with 720 DOF to obtain the numerical wavenumbers and wave modes, and the numerical spectral matrix for the whole structure uses 96 DOF in the rod case and 192 DOF for the other beam cases. Latter, in the condensed case, these models are reduced to 4 DOF, in the beam cases, and 2 DOF, in the rod case. This indicates that the WSFEM is an alternative to conventional finite element analysis requiring much less computational effort for solving harmonic problems for long structural waveguides.

It is important to point out that numerical problems still occur when higher order propagation modes are included in the formulation presented in this paper. Despite this handicap, when applying an off-centered axial excitation, the proposed approach provided, to some extent, information about the local effects induced by this type of excitation. Future work is needed to improve the WSFEM formulation in order to provide solutions for the numerical problems that are still encountered.

\section{Acknowledgments}

The authors are grateful to the government funding agency Fundação de Amparo à Pesquisa de São Paulo, FAPESP, for the financial support for the present research work through processes 2009/10715-1 and 2010/17317-9.

\section{References}

[1] B. Aalami, Waves in prismatic guides of arbitrary cross section, Journal of Applied Mechanics - ASME 40 (1973), 1067-1072.

[2] J.R.F. Arruda and R.F. Nascimento, Building spectral element dynamic matrices using finite element models of waveguide slices, in: Proceeding of the Noise and Vibration: Emerging Methods, Oxford, 2009.

[3] J.R.F. Arruda and R.F. Nascimento, Building spectral elements from finite element models of waveguide slices, in: Proceedings of the Ninth International Conference on Computational Structures Technology, Stirlingshire, Civil-Comp Press, 9 (2008).

[4] J.R.F. Arruda, K.M. Ahmida, M.N. Ichchou and J.M. Mencik, Investigating the relations between the wave finite element and spectral element methods using simple waveguides, in: Proceedings of the 19th International Congress of Mechanical Engineering, Brasília, ABCM, 2007.

[5] F. Benmeddour, F. Treyssède and L. Laguerre, Numerical modeling of guided wave interaction with non-axisymmetric cracks in elastic cylinders, International Journal of Solids and Structures 48 (2011), 764-774.

[6] J.S. Bolton, H.J. Song, Y.K. Kim and D.E. Newland, The wave number decomposition approach to the analysis of tire vibration, in: Proceedings of Noise-Con 98, Ypsilanti, 97 (1998).

[7] J.C. Delamotte, R.F. Nascimento and J.R.F. Arruda, Simple models for the dynamic modeling of rotating tires, Shock and Vibration 15 (2008), 383-393.

[8] S.B. Dong and R.B. Nelson, On natural vibrations and waves in laminated orthotropic plates, Journal of Applied Mechanics 39 (1972), 739-745. 
[9] J.F. Doyle, Wave Propagation in Structures, Springer-Verlag, New York, 1989, 2/E 1997.

[10] S. Finnveden, Exact spectral finite element analysis of stationary vibrations in a railway car structure, Acta Acustica 2 (1994), 433-449.

[11] L. Gavric, Computation of propagative waves in free rail using a finite element technique, Journal of Sound and Vibration 185 (1995), $531-543$.

[12] L. Gavric, Finite element computation of dispersion properties of thin-walled waveguides, Journal of Sound and Vibration 173 (1994), $113-124$.

[13] G.H. Golub and C.F.V. Loan, Matrix Computations, The John Hopkins University Press, 1996.

[14] L. Hinke, B.R. Mace and M.J. Brennan, Finite element analysis of waveguides, in: ISVR Technical Memorandum 932 (2004).

[15] M.N. Ichchou, S. Akrouta and J.-M. Mencik, Guided waves group and energy velocities via finite elements, Journal of Sound and Vibration 305 (2007), 931-944.

[16] P. Loveday, Simulation of piezoelectric excitation of guided waves using waveguide finite elements, IEEE Transactions on Ultrasonics, Ferroelectrics and Frequency Control 55 (2008), 2038-2045.

[17] B.R. Mace, D. Duhamel, M.J. Brennan and L. Hinke, Finite element prediction of wave motion in structural waveguides, J Acoust Soc Am 117 (2005), 5, 2835-2843.

[18] A. Marzani, Time-transient response for ultrasonic guided waves propagating in damped cylinders, International Journal of Solids and Structures 45 (2008), 6347-6368.

[19] D.J. Mead, A general theory of harmonic wave propagation in linear periodic systems with multiple coupling, Journal of Sound and Vibration 27 (1973), 2, 235-260.

[20] J.-M. Mencik, On the low- and mid-frequency forced response of elastic structures using wave finite elements with one-dimensional propagation, Computers and Structures 88 (2010), 674-689.

[21] J.-M. Mencik and M.N. Ichchou, Multi-mode propagation and diffusion in structures through finite elements, European Journal of Mechanics A/Solids 24 (2005), 877-898.

[22] W.D. Pilkey, Analysis and Design of Elastic Beams: Computational Methods, John Wiley \& Sons, New York, 2002.

[23] P.B. Silva, J.R.F. Arruda and A. Goldstein, Study of elastic band-gaps in finite periodic structure using finite element models, in: Proceeding of the 15th International Symposium on Dynamic Problems in Mechanics, vol. 118, São Sebastião, A.T. Fleury and P.R.G. Kurka, eds, ABCM, 2011.

[24] Y. Waki, B.R. Mace and M.J. Brennan, Numerical issues concerning the wave and finite element method for free and forced vibrations of waveguides, Journal of Sound and Vibration 327 (2009), 92-108.

[25] X. Zhong and F.W. Williams, On the direct solution of wave propagation for repetitive structures, Journal of Sound and Vibration 181 (1995), 485-501. 

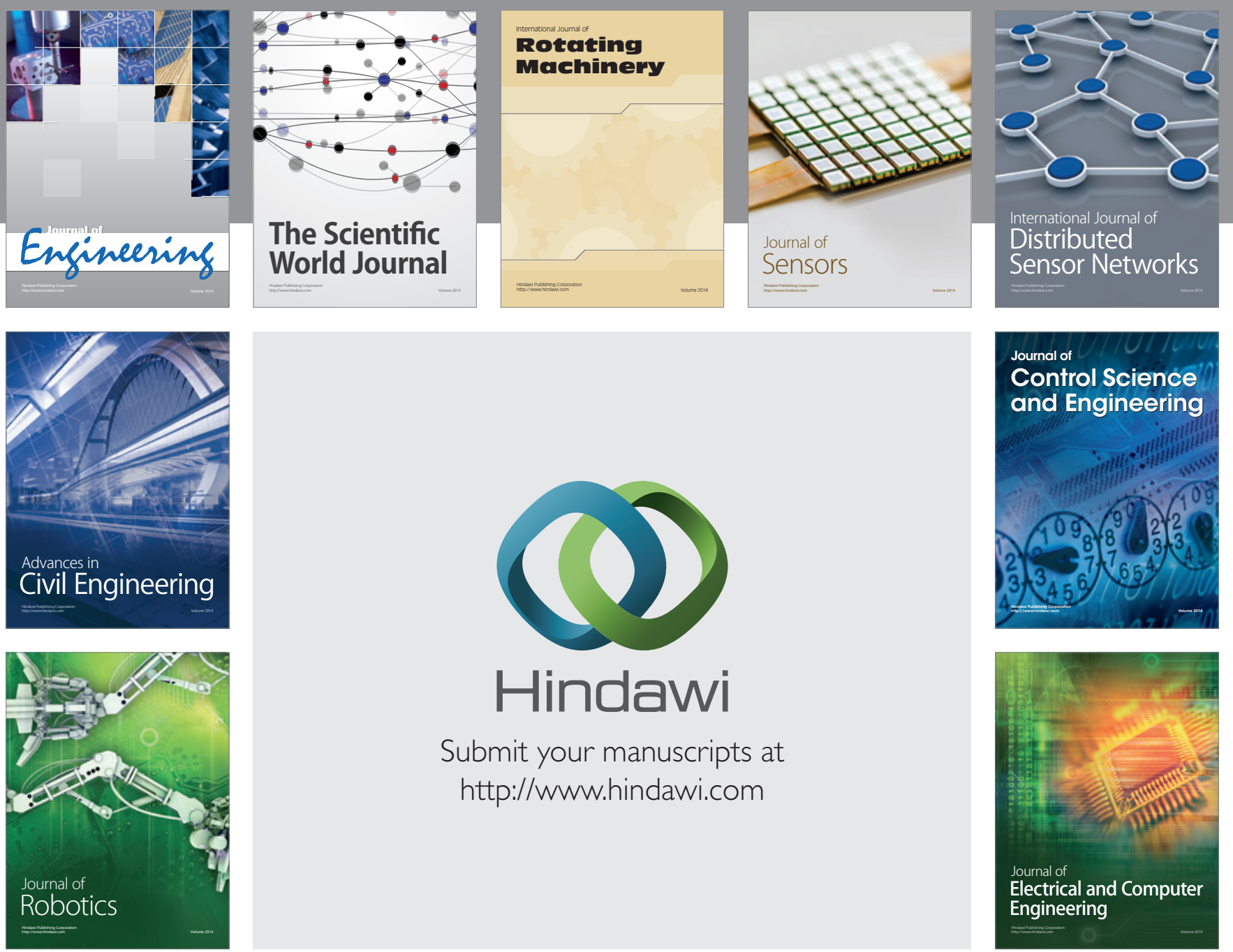

Submit your manuscripts at

http://www.hindawi.com
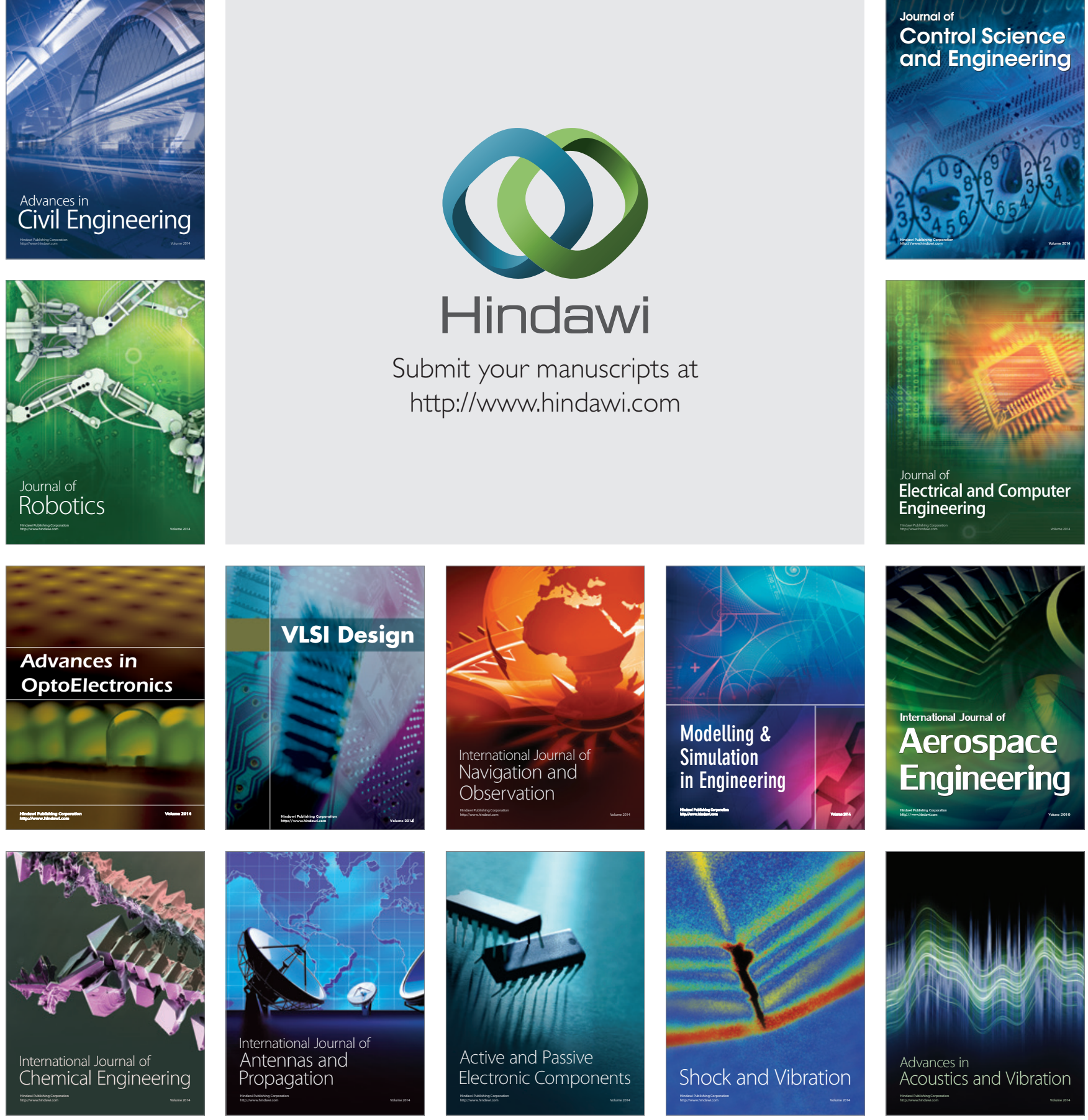